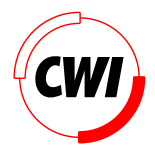

Centrum voor Wiskunde en Informatica REPORTRAPPORT

Remarks on food chain dynamics

Yu.A. Kuznetsov and S. Rinaldi

Department of Analysis, Algebra and Geometry

AM-R9513 1995 
Report AM-R9513

ISSN 0924-2953

CWI

P.O. Box 94079

1090 GB Amsterdam

The Netherlands

$\mathrm{CWI}$ is the National Research Institute for Mathematics and Computer Science. CWI is part of the Stichting Mathematisch Centrum (SMC), the Dutch foundation for promotion of mathematics and computer science and their applications.

SMC is sponsored by the Netherlands Organization for Scientific Research (NWO). CWI is a member of ERCIM, the European Research Consortium for Informatics and Mathematics.

Copyright (C) Stichting Mathematisch Centrum P.O. Box 94079, 1090 GB Amsterdam (NL) Kruislaan 413, 1098 SJ Amsterdam (NL) Telephone +3120 5929333 Telefax +31205924199 


\title{
Remarks on Food Chain Dynamics
}

\author{
Yu.A. Kuznetsov*, S. Rinaldi ${ }^{\dagger}$ \\ * Institute of Mathematical Problems of Biology, \\ Russian Academy of Sciences, Pushchino, Moscow Region, \\ 142292 Russia \\ $\&$ \\ CWI, \\ P.O. Box 94079, 1090 GB Amsterdam, The Netherlands \\ † Centro Teoria dei Sistemi, Consiglio Nazionale delle Ricerche, \\ Politecnico di Milano, \\ via Ponzio 34/5, 20133 Milano, \\ Italy
}

\begin{abstract}
The main modes of behavior of a food chain model, composed of logistic prey and Holling type II predator and superpredator, are discussed in this paper. The study is carried out through bifurcation analysis, alternating between a normal form approach and numerical continuation. The two-parameter bifurcation diagram of the model contains Hopf, fold and transcritical bifurcation curves of equilibria as well as flip, fold and transcritical bifurcation curves of limit cycles. The appearance of chaos in the model is proved to be related to a Hopf bifurcation and a degenerate homoclinic bifurcation in the prey-predator subsystem. The boundary of the chaotic region is shown to have a very peculiar structure.
\end{abstract}

AMS Subject Classification (1991): 34C05, 34C28, 65L07, 92A15

Keywords \& Phrases: Food chain, mathematical models, bifurcations, normal forms, chaos

Note: The paper is submitted for publication to Mathematical Biosciences

\section{INTRODUCTION}

The aim of this paper is to classify the asymptotic modes of behavior of three trophic-level food chains composed by a prey $(X)$, a predator $(Y)$ and a superpredator $(Z)$. This basic and classical problem in population dynamics has been dealt with by many authors. Rescigno and Jones [30] were perhaps the first to handle the problem using a general mathematical model of the form:

$$
\left\{\begin{aligned}
\dot{X} & =X \mathcal{F}(X, Y) \\
\dot{Y} & =Y \mathcal{G}(X, Y, Z) \\
\dot{Z} & =Z \mathcal{H}(Y, Z) .
\end{aligned}\right.
$$

Their analysis, mainly concerned with oscillations and cycles, was a bit naive and, indeed, their results do not agree with many of the subsequent findings. 
A rich literature exists on the problem of persistence of food chains in all its versions: weak persistence (i.e. $\lim \sup >0$ for all populations) $[8,10,12]$; strong persistence (i.e. $\lim \inf >0$ for all populations) [6,9]; and uniform persistence (i.e. $\lim \inf \geq \varepsilon>0$ for all populations) [7, 11]. Since the superpredator of a food chain goes extinct if one of the other populations does so, persistence only depends on the behavior of the system in the vicinity of the plane $Z=0$, i.e. the face $(X, Y)$ of the state space. More precisely, the populations persist if the injection of a small number of superpredators gives rise to the invasion into the positive octant $(\dot{Z}>0)$ from a positive equilibrium $x^{0}$ or from a limit cycle $L_{0}$ lying on the face $Z=0$. Thus, as noted by Freedman and Waltman [8], a food chain is persistent if the function $\mathcal{H}$ in (1.1) evaluated at $x^{0}$ is positive, or if the average value of $\mathcal{H}$ along $L_{0}$ is positive. Obviously, the explicit conditions derived from the positivity of $\mathcal{H}$, or through a more elegant and effective technique based on Lyapunov-like persistence functions $[10,11,12]$, do not provide information on the number and structure of the attractors in the positive octant. This is a very unfortunate situation, because the type of transients and the nature of the attractors are, after all, the most interesting properties of a dynamical system.

More insights were obtained by Muratori [23] and by Muratori and Rinaldi [26, 27] who applied the singular perturbation technique to food chains with trophic levels exhibiting diversified time responses. In particular, they derived explicit conditions for the persistence of the three populations and for the

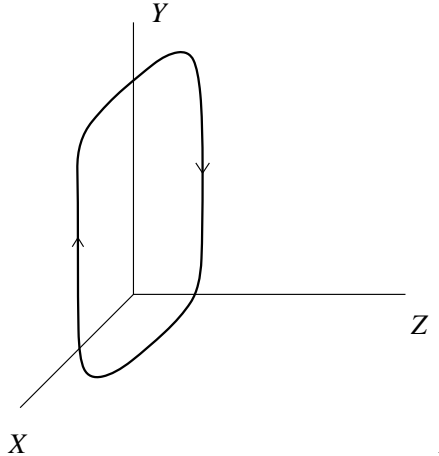

(a)

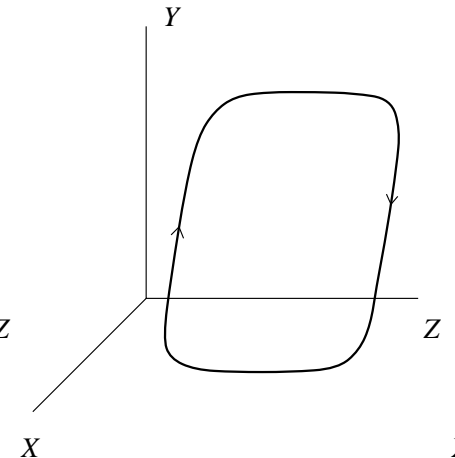

(b)

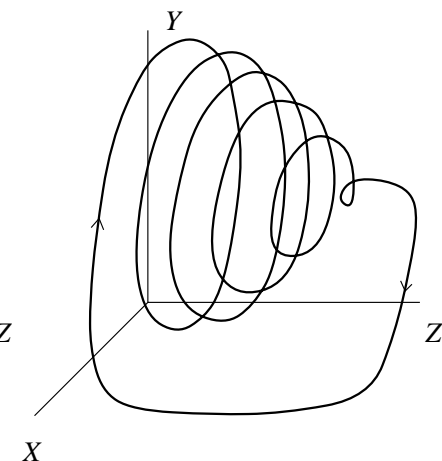

(c)

Figure 1.1: Limit cycles in a food chain composed of prey $(X)$, predator $(Y)$ and superpredator $(Z)$ : (a) high-frequency cycle (prey-predator interaction); (b) low-frequency cycle (predator-superpredator interaction); (c) low-frequency cycle with a burst of high-frequency oscillations.

existence of limit cycles. Moreover, their analysis shows that, if the time responses are increasing with the trophic level, the limit cycles can be of three distinct types: high-frequency cycles due to preypredator interactions (Figure 1.1(a)); low-frequency cycles due to predator-superpredator interactions (Figure 1.1(b)); and very special low-frequency cycles, called "tea cup" limit cycles (Figure 1.1(c)), containing prey-predator oscillations during a fraction of their period. The last is obviously the most interesting case, in which the dynamics of the fastest component of the system, the prey, is not completely filtered out and is periodically revealed by a burst of high-frequency oscillations. Similar dynamics has also been found in models describing electrical activity in pancreatic cells $[4,33]$ and dynastic cycles in ancient China [28]. For a theoretical discussion on bursting phenomena see Terman $[34,35]$.

Many simulation studies have also shown that food chain models can have chaotic dynamics, generally obtained through a cascade of period doublings $[1,2,14,15,21,22,29,31,36]$. The most interesting of these studies performed by Hastings and Powell [14] shows that food chains characterized by time responses increasing with the trophic level have strange attractors which resemble very much the cycle in Figure 1.1(c), and are, therefore, called "tea-cup" strange attractors. Not all parameter values used in the above mentioned papers are biologically meaningful (see [21]) but some of them are, like those used by Scheffer [31] for plankton and by Wilder et al. [36] for a study on gypsy moths. This fact, together with the analysis carried out by Abrams and Roth $[2,1]$ and by 
McCann and Yodzis [21] on food chains composed of vertebrates and invertebrates, strongly supports the conjecture that the irregular dynamics observed in many natural food chains and food webs might, indeed, be that of a strange attractor.

The above results justify the interest for a deeper understanding of the complexity of food chains, which naturally calls for bifurcation analysis of food chain models. This has been done in two recent papers devoted to the standard food chain (logistic prey and Holling type II predator and superpredator). In the first paper, Klebanoff and Hastings [17] performed a theoretical analysis by deriving the normal form of a degenerate bifurcation point (called point $M$ from now on) and by showing that this normal form might imply chaos for small perturbations of the parameters. This is conceptually related to the above mentioned studies on persistence, because their degenerate bifurcation point corresponds to parameter values giving rise to the following two singularities:

(i) the limit cycle $L_{0}$ shrinks to the equilibrium $x^{0}$ on the $(X, Y)$ face;

(ii) the function $\mathcal{H}$ is zero at $x^{0}$, so that invasion from $x^{0}$ is neither guaranteed nor excluded.

In the second paper, McCann and Yodzis [22] performed mainly numerical analyses of the bifurcations of the model by means of simulations and continuation. They started from the same degenerate point $M$ but showed that chaos arises in a different region of the parameter space. This discrepancy between the normal form analysis and the numerical results is also evident in [17], where all simulations showing strange attractors refer to parameter values far from those characterizing the degenerate point.

This paper is devoted to a more comprehensive bifurcation analysis of the standard food chain model. In the next section, the model is described and the relationships among parameters giving rise to the two degeneracies $(i)$ and $(i i)$ are identified. In Section 3, the normal form of the degenerate point is revisited and used to derive, by numerical continuation and further normal form analysis, a detailed picture of the bifurcation structure. This picture includes Hopf, fold and transcritical bifurcations of equilibria, as well as fold, flip and transcritical bifurcations of cycles, and shows that chaos is obtained via a cascade of limit cycle bifurcations in a region of the parameter space that is far from the degenerate point. This definitely proves that the origin of chaos in standard food chain systems cannot be associated to point $M$, but rather to more complex and global dynamical phenomena. Indeed, the singular-perturbation analysis of the food chain with diversified time responses, as presented in Section 4 , indicates that chaos is related to the creation of a tea-cup attractor due to a singular Hopf bifurcation and to its destruction via a double homoclinic bifurcation, which has the consequence of cutting this attractor into two pieces. These are key features for a better understanding of the dynamics of food chains, as pointed out in the last section, where some general interpretations of the results are given. The details of the normal form derivation are confined to the Appendix.

\section{THE MODEL}

The food chain we analyse in this paper is the standard food chain composed of a logistic prey $(X)$, a Holling type II predator $(Y)$ and a Holling type II superpredator $(Z)$. It is, therefore, modelled by

$$
\begin{aligned}
& \frac{d X}{d T}=X\left(R\left(1-\frac{X}{K}\right)-\frac{A_{1} Y}{B_{1}+X}\right), \\
& \frac{d Y}{d T}=Y\left(E_{1} \frac{A_{1} X}{B_{1}+X}-D_{1}-\frac{A_{2} Z}{B_{2}+Y}\right), \\
& \frac{d Z}{d T}=Z\left(E_{2} \frac{A_{2} Y}{B_{2}+Y}-D_{2}\right),
\end{aligned}
$$

where $T$ is time, $R$ and $K$ are intrinsic growth rate and carrying capacity of prey, the $A_{i}$ 's are maximum predation rates, the $B_{i}$ 's are half saturation constants, the $D_{i}$ 's are death rates and the $E_{i}$ 's are efficiencies of predator $(i=1)$ and superpredator $(i=2)$. Obviously, we assume that $E_{i} A_{i}>D_{i}, i=1,2$, in order to avoid the case where predator and superpredator cannot survive even when their food is infinitely abundant. 
In order to obtain simpler analytical forms, we rescale the variables following Klebanoff and Hastings $[17]$

$$
x_{1}=\frac{X}{K}, x_{2}=\frac{Y}{K E_{1}}, x_{3}=\frac{Z}{K E_{1} E_{2}}, t=R T
$$

thus obtaining

$$
\begin{aligned}
\frac{d x_{1}}{d t} & =x_{1}\left(1-x_{1}-\frac{a_{1} x_{2}}{1+b_{1} x_{1}}\right), \\
\frac{d x_{2}}{d t} & =x_{2}\left(\frac{a_{1} x_{1}}{1+b_{1} x_{1}}-d_{1}-\frac{a_{2} x_{3}}{1+b_{2} x_{2}}\right), \\
\frac{d x_{3}}{d t} & =x_{3}\left(\frac{a_{2} x_{2}}{1+b_{2} x_{2}}-d_{2}\right),
\end{aligned}
$$

where

$$
a_{1}=\frac{K A_{1} E_{1}}{R B_{1}}, b_{1}=\frac{K}{B_{1}}, d_{1}=\frac{D_{1}}{R}, a_{2}=\frac{K A_{2} E_{1} E_{2}}{R B_{2}}, b_{2}=\frac{K E_{1}}{B_{2}}, d_{2}=\frac{D_{2}}{R} .
$$

Then, the above necessary conditions for the persistence of predator and superpredator become $a_{i}>$ $b_{i} d_{i}, i=1,2$.

Table 1 contains the numerical values of the parameters used by different authors to perform simulations with model (2.4)-(2.6) or with very similar models. The parameter values used in this paper, sometimes called the reference values, are the following

$$
a_{1}=5, a_{2}=0.1, b_{1}=3, b_{2}=2,
$$

while $d_{1}$ and $d_{2}$ are varied.

All coordinate axes and faces of the positive octant are invariant sets of system (2.4)-(2.6). Moreover, there are three trivial equilibria:

- the origin $(0,0,0)$, which is always a saddle;

- the point $(1,0,0)$, corresponding to prey at carrying capacity and absence of predator and superpredator;

- the point

$$
x^{0}=\left(x_{1}^{0}, x_{2}^{0}, 0\right)=\left(\frac{d_{1}}{a_{1}-b_{1} d_{1}}, \frac{a_{1}-d_{1}\left(b_{1}+1\right)}{\left(a_{1}-b_{1} d_{1}\right)^{2}}, 0\right),
$$

\begin{tabular}{|l||l|l|l|l|l|l|}
\hline & \multicolumn{1}{|c|}{$a_{1}$} & \multicolumn{1}{|c|}{$a_{2}$} & \multicolumn{1}{|c|}{$b_{1}$} & \multicolumn{1}{|c|}{$b_{2}$} & $d_{1}$ & $d_{2}$ \\
\hline \hline Hogeweg and Hesper [15] & 1.81 & 0.181 & $4.5^{*}$ & $0.45^{*}$ & 0.16 & 0.08 \\
\hline Scheffer [31] & 8.0 & 2.88 & 6.66 & 2.4 & 0.87 & 0.25 \\
\hline Hastings and Powell [14] & 5.0 & 0.1 & $4.0^{\dagger}$ & 2.0 & 0.4 & 0.01 \\
\hline Rai and Sreenivasan [29] & 0.22 & 0.011 & 5.0 & 0.25 & 0.02 & 0.02 \\
\hline Abrams and Roth [1] (ex.3) & $1.75^{\dagger}$ & $0.35^{\dagger}$ & $2.62^{\dagger}$ & $1.75^{\dagger}$ & 0.1 & 0.04 \\
\hline Abrams and Roth [1] (ex.4) & $2.5^{\dagger}$ & $0.25^{\dagger}$ & $3.0^{\dagger}$ & $2.0^{\dagger}$ & 0.2 & 0.025 \\
\hline McCann and Yodzis [22] & 2.4 & 0.022 & 2.98 & 0.44 & 0.4 & 0.01 \\
\hline
\end{tabular}

Table 2.1: Numerical values of the six parameters of the model (2.4)-(2.6) used by different authors to simulate the behavior of food chains. Values indicated by ${ }^{*}$ are estimates (because the functional form of the model is slightly different from (2.4)-(2.6)). Values indicated by ${ }^{\dagger}$ are the center values of the range used in the simulations. 
which is positive for $a_{1}>d_{1}\left(b_{1}+1\right)$ and corresponds to prey-predator coexistence and absence of superpredator.

The point $x^{0}$ can be either stable or unstable in the face $\left(x_{1}, x_{2}\right)$. When it is unstable, it is surrounded by a stable limit cycle [20], which is unique and globally attracting in the plane $x_{3}=0$ [5]. The transition between the two situations, corresponding to degeneracy $(i)$ mentioned in the preceding section, is a supercritical Hopf bifurcation of the submodel (2.4)-(2.5) with $x_{3}=0$, taking place at

$$
b_{1} d_{1}\left(b_{1}+1\right)=a_{1}\left(b_{1}-1\right)
$$

(note that $b_{1}>1$ if the carrying capacity $K$ is greater than the half saturation constant $B_{1}$ ). Moreover, the second degeneracy of the point $x^{0}$ (condition ( $\left.i i\right)$ above) takes place at

$$
\left(a_{1}-b_{1} d_{1}\right)^{2} d_{2}=\left(a_{1}-d_{1}\left(1+b_{1}\right)\right)\left(a_{2}-b_{2} d_{2}\right) .
$$

It is a transcritical bifurcation giving rise to a strictly positive equilibrium for small perturbations of the parameters.

The bifurcation analysis of (2.4)-(2.6) will be carried out with respect to two parameters. Although, in principle, any pair of parameters might be selected, the results will be derived only for the pair $\left(d_{1}, d_{2}\right)$ for two independent reasons. First, because the death rates $D_{1}$ and $D_{2}$ in (2.1)-(2.3) (and, hence, $d_{1}$ and $d_{2}$ in (2.4)-(2.6)) can be strongly influenced, for example by harvesting the predator and the superpredator or by contaminating or reclaiming their environments, so that this pair of parameters is the most interesting one for interpreting and forecasting the consequences of management actions. Second, because $d_{1}$ and $d_{2}$ influence, separately, only one state equation, thus facilitating singular perturbation analysis. This does not mean that other bifurcation diagrams are of no interest. For example, the effects of enrichment of food chain systems, recently discussed in $[1,2]$ through simulations, could certainly be studied in a more compact form by producing bifurcation diagrams of the model (2.1)-(2.3) with respect to $R$ and $K$.

\section{BIFURCATION ANALYSIS}

The analysis starts from the point $M$ giving rise to the singularities $(i)$ and $(i i)$. This is a point in the $\left(d_{1}, d_{2}\right)$-plane at which equations $(2.8)$ and (2.9) are satisfied, so that the corresponding Jacobian matrix has one zero eigenvalue and two purely imaginary eigenvalues. The parameter-dependent normal form of the system near this point will be derived and used to show that, for meaningful values of the other parameters, five bifurcation curves emerge from this point, none of which implies chaos. Then, these curves will be continued in the parameter plane $\left(d_{1}, d_{2}\right)$ by means of LOCBIF [16], an interactive software implementing a powerful continuation technique in conjunction with detection of high codimension bifurcation points. The normal form approach will be used to find out how many and which bifurcation curves are rooted at each one of these high-codimension points. By alternating these two phases (continuation and normal form analysis), the bifurcation structure will emerge and indicate where strange attractors can be found in the parameter plane.

\subsection{Normal form of codimension-two point $M$}

If all parameters, except $d_{1}$ and $d_{2}$, are fixed, the planar Hopf bifurcation and the transcritical bifurcation occur along two curves in the $\left(d_{1}, d_{2}\right)$-plane, given by (2.8) and (2.9) and labeled by $H_{p}$ and $T C_{e}$ in the following figures. These curves intersect at the point $M$ with coordinates

$$
d^{*}=\left(d_{1}^{*}, d_{2}^{*}\right)=\left(\frac{a_{1}\left(b_{1}-1\right)}{b_{1}\left(b_{1}+1\right)}, \frac{a_{2}\left(b_{1}+1\right)^{2}}{b_{2}\left(b_{1}+1\right)^{2}+4 a_{1} b_{1}}\right) .
$$

The coordinates (see (2.7)) of the corresponding equilibrium point $x^{0}$ are

$$
x^{*}=\left(x_{1}^{*}, x_{2}^{*}, x_{3}^{*}\right)=\left(\frac{b_{1}-1}{2 b_{1}}, \frac{\left(b_{1}+1\right)^{2}}{4 a_{1} b_{1}}, 0\right) .
$$


The analysis of the bifurcations in the vicinity of $x^{*}$ for parameter values close to $d^{*}$ can be performed using the normal form technique $[3,13]$. But the well-known results on bifurcations of equilibria with a zero eigenvalue and two purely imaginary eigenvalues can not be merely applied here, since the dynamical system (2.4)-(2.6) is not generic. Its functional form, as shown below, leads to the existence of a tangent (fold) bifurcation curve $T_{e}$ passing through $M$. As noticed by McCann and Yodzis [22], the existence of the two bifurcation curves $T_{e}$ and $T C_{e}$ is rather peculiar, because only the curve $T_{e}$ is expected in general, while only the curve $T C_{e}$ should be present in generic systems with invariant coordinate planes.

The normal form analysis of (2.4)-(2.6) has already been carried out by Klebanoff and Hastings [17]. However, it can be improved in two respects. First of all, it is possible to perform a parameterdependent normalization near the degenerate point $M$. Moreover, generically, the number of nonlinear terms in the normal form can be reduced by a nonlinear time reparametrization. The normalization and reparametrization procedures are described in the Appendix. The result is that system (2.4)-(2.6) near point $x^{*}$ for parameter values close to $d^{*}$ can generically be written in the normal form

$$
\left\{\begin{array}{l}
\dot{\eta}=\beta \eta+\gamma \eta^{2}+A_{1} \rho^{2} \eta+B \eta^{3}+O\left(\|(\eta, \rho)\|^{4}\right) \\
\dot{\rho}=\alpha \rho+C_{1} \eta \rho+E \rho^{3}+O\left(\|(\eta, \rho)\|^{4}\right) \\
\dot{\theta}=\omega+O(\|(\eta, \rho)\|)
\end{array}\right.
$$

where $\eta$ is a coordinate in the $x_{3}$-direction, while $(\rho, \theta)$ are polar coordinates in the plane $x_{3}=0$. At $d=d^{*}$, this normal form differs from that derived by Klebanoff and Hastings [17], who, indeed did not reparametrize time. For this reason, their normal form contains also an $\eta^{2} \rho$-term in the second equation. In (3.1) $\alpha, \beta, \gamma$ are functions of the parameters $\left(d_{1}, d_{2}\right)$ and vanish at $d=d^{*}$, while $\omega, A_{1}, B, C_{1}$ and $E$ do not vanish, in general, at $d=d^{*}$. Explicit formulas for $\alpha, \beta$ and $\gamma$, can be found in the Appendix, together with those (already obtained by Klebanoff and Hastings) for $\omega, A_{1}, B, C_{1}$ and $E$ at the critical parameter values. It can also be shown that the Jacobian matrix of the map $\left(d_{1}, d_{2}\right) \mapsto(\alpha, \beta)$ is nonsingular at $d=d^{*}$. Therefore, one can consider $(\alpha, \beta)$ as new coordinates in the parameter plane with their origin at $d^{*}$, namely,

$$
\begin{aligned}
\alpha & =-\frac{b_{1}^{2}-1}{4 a_{1}}\left(d_{1}-d_{1}^{*}\right)+O\left(\left\|d-d^{*}\right\|^{2}\right), \\
\beta & =-\left(d_{2}-d_{2}^{*}\right)+O\left(\left\|d-d^{*}\right\|^{2}\right),
\end{aligned}
$$

and treat all other coefficients involved in (3.1) as functions of $(\alpha, \beta)$. It follows from the parameterdependent normalization that the coefficient $\gamma$ in the first equation cannot be considered as an independent parameter, as it was in [17]. Actually, it has the form

$$
\gamma(\alpha, \beta)=\gamma_{1} \alpha-\gamma_{2} \beta+O\left(\|(\alpha, \beta)\|^{2}\right),
$$

where $\gamma_{1,2}>0$ are suitable constants (see Appendix). It is this fact that gives rise to the existence of both bifurcation curves $T_{e}$ and $T C_{e}$.

Truncating terms of order four and higher in $(\eta, \rho)$ in $(3.1)$, one obtains a system in which the first two equations are independent of $\theta$,

$$
\left\{\begin{array}{l}
\dot{\eta}=\beta \eta+\gamma \eta^{2}+A_{1} \rho^{2} \eta+B \eta^{3} \\
\dot{\rho}=\alpha \rho+C_{1} \eta \rho+E \rho^{3}
\end{array}\right.
$$

while the third equation describes an azimuthal rotation with almost constant velocity.

Equilibria of (3.2) with $\rho=0$ and $\eta>0$ represent strictly positive equilibria of the original system (2.4)-(2.6), while equilibria of (3.2) with $\eta=0$ and $\rho>0$ describe limit cycles in the invariant plane $x_{3}=0$. Finally, a strictly positive equilibrium of (3.2) corresponds to a strictly positive limit cycle in the original system (2.4)-(2.6), while a limit cycle in (3.2) would correspond to a torus or to a more complex invariant set of (2.4)-(2.6). Thus, bifurcations of the equilibria in (3.2) correspond to 
bifurcations of equilibria and cycles in (2.4)-(2.6). For example, a transcritical bifurcation of a nonzero equilibrium on the $\eta$-axis of system (3.2) corresponds to the Hopf bifurcation of a strictly positive equilibrium in (2.4)-(2.6).

The bifurcation diagram of the normal form (3.2) near the origin of the $(\alpha, \beta)$-plane depends on the signs of the coefficients $A_{1}, B, C_{1}$ and $E$ at $\alpha=\beta=0$, as well as upon $\gamma_{1}$. However, as can be seen from the Klebanoff-Hastings formulae, the coefficients $A_{1}, B$ and $E$ are negative for all values of the parameters. Therefore, only two general cases are possible, depending on the sign of the coefficient $C_{1}$. This conclusion differs from that of Klebanoff and Hastings [17] who considered more cases than those actually occurring. In the following, only the case $C_{1}<0$ is considered, because all the parameter settings reported in Table 1 fall into this category, as can be easily verified using the Klebanoff-Hastings formula for $C_{1}$ (see Appendix).

In the considered case, the system (3.2) can be further simplified by rescaling its variables, parameters and time. In fact, the substitution

$$
\eta \mapsto \frac{C_{1}}{B} \eta, \rho \mapsto-\frac{C_{1}}{\sqrt{B E}} \rho, \alpha \mapsto-\frac{C_{1}^{2}}{B} \alpha, \beta \mapsto-\frac{C_{1}^{2}}{B} \beta, \quad t \mapsto-\frac{B}{C_{1}^{2}} t
$$

reduces $(3.2)$ to

$$
\left\{\begin{array}{l}
\dot{\eta}=\beta \eta+\left[\Gamma_{1} \alpha-\Gamma_{2} \beta+O\left(\|(\alpha, \beta)\|^{2}\right)\right] \eta^{2}+A_{2} \rho^{2} \eta-\eta^{3} \\
\dot{\rho}=\alpha \rho-\eta \rho-\rho^{3}
\end{array}\right.
$$

where the dots now mean differentiation with respect to the new time, and

$$
A_{2}=-\frac{A_{1}}{E}, \Gamma_{k}=\frac{C_{1}}{B} \gamma_{k}, k=1,2
$$

The bifurcation diagram of the normal form (3.3) (see Figure 3.1) can be interpreted in terms of bifurcations of the original system (2.4)-(2.6). Point $M$ (origin of the $(\alpha, \beta)$-plane) is the root of five bifurcation curves:

- the planar Hopf

$$
H_{p}=\{(\alpha, \beta): \alpha=0\}
$$

- the transcritical of equilibria

$$
T C_{e}=\{(\alpha, \beta): \beta=0\},
$$

- the tangent (fold) of positive equilibria

$$
T_{e}=\left\{(\alpha, \beta): \beta=-\frac{1}{4} \Gamma_{1}^{2} \alpha^{2}+O\left(\alpha^{3}\right), \alpha>0\right\},
$$

- the Hopf of positive equilibria

$$
H=\left\{(\alpha, \beta): \beta=\left(1-\Gamma_{1}\right) \alpha^{2}+O\left(\alpha^{3}\right), \alpha>0\right\},
$$

- the transcritical of limit cycles

$$
T C_{c}=\left\{(\alpha, \beta): \beta=-A_{2} \alpha, \alpha>0\right\} .
$$

If

$$
4\left(\Gamma_{1}-1\right)<\Gamma_{1}^{2}
$$

(as it is for the reference values of the parameters), the Hopf bifurcation curve $H$ is located between the transcritical curve $T C_{e}$ and the fold bifurcation curve $T_{e}$. To understand bifurcations of the system 


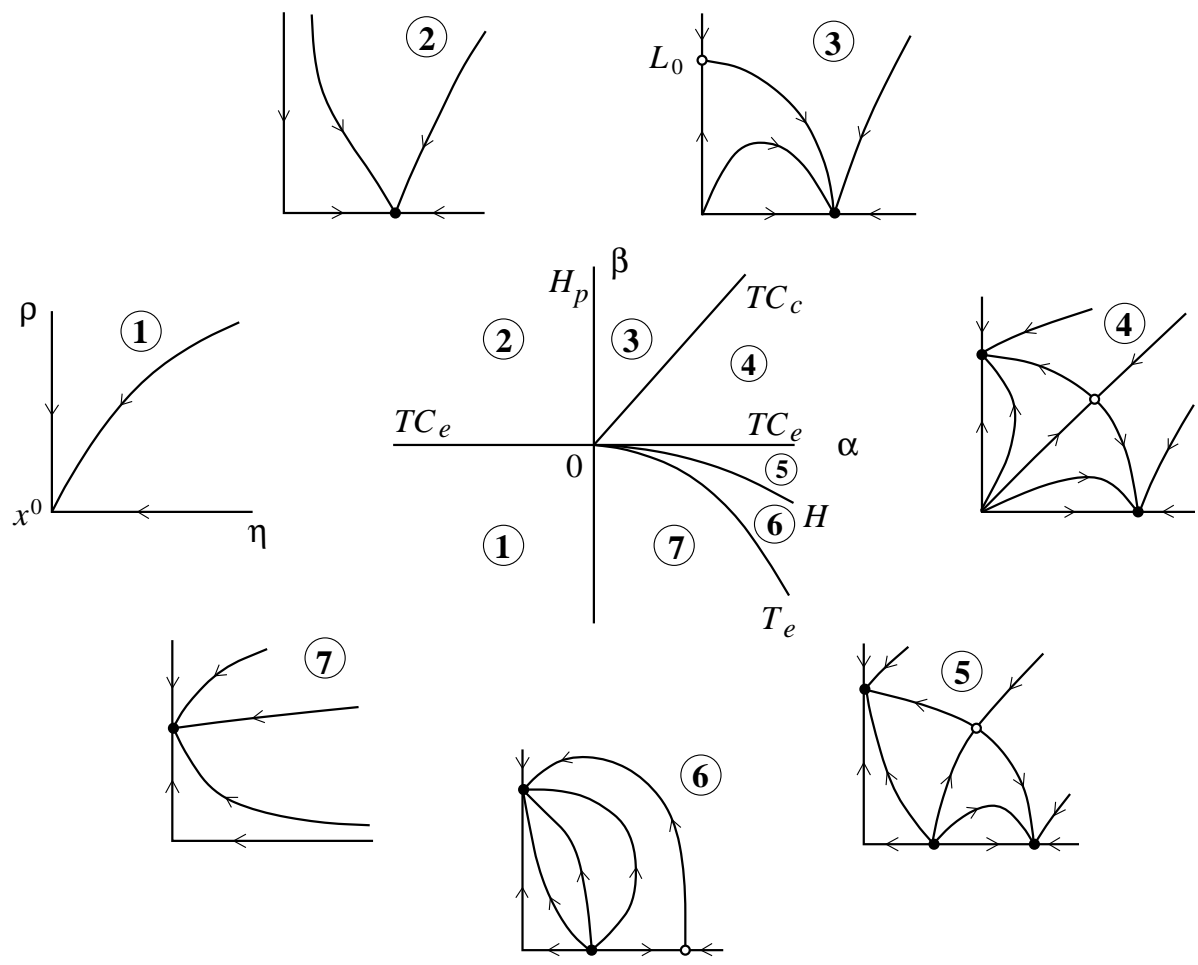

Figure 3.1: Bifurcation diagram and corresponding phase portraits of the normal form (3.3) in the vicinity of the codimension-two point $M$.

(2.4)-(2.6) corresponding to the diagram presented in Figure 3.1, a clockwise trip around the origin of the parameter plane is useful.

In region $\mathbf{1}$, the equilibrium $x^{0}$ on the face $x_{3}=0$ is stable and is the unique attractor of the system. Crossing the boundary $T C_{e}$ of regions $\mathbf{1}$ and $\mathbf{2}, x^{0}$ becomes a saddle and a stable strictly positive equilibrium emerges from $x^{0}$ via the transcritical bifurcation. Then, at the boundary $H_{p}$ separating regions 2 and $\mathbf{3}$, the equilibrium $x^{0}$ becomes a repellor and a saddle limit cycle $L_{0}$ appears on the face $x_{3}=0$ (planar Hopf bifurcation). The transition to region 4 through the line $T C_{c}$ implies a transcritical bifurcation of this cycle, which becomes stable, and leads to the appearance of a positive saddle cycle. Then, a second strictly positive equilibrium appears in (2.4)-(2.6) on the line $T C_{e}$, so that in region 5 there exist two strictly positive equilibria (only one of which is stable), a stable planar cycle and a strictly positive saddle cycle. At the boundary $H$ between regions 5 and $\mathbf{6}$, the saddle limit cycle shrinks to the equilibrium and disappears via a Hopf bifurcation. Therefore, the planar cycle $L_{0}$ is the only attractor in region 6 . Then, the two positive equilibria collide and disappear on the curve $T_{e}$ separating region 6 from region $\mathbf{7}$, and finally the planar cycle $L_{0}$ shrinks to $x^{0}$ on the planar Hopf bifurcation curve $H_{p}$.

So far, no chaotic behavior has been detected near the codimension-two point $M$ in the truncated system. Since system (3.3) has no limit cycles, then system (2.4)-(2.6) cannot behave chaotically near point $x^{0}$ for $d$ close to $d^{*}$. This means that the codimension-two point $M$ can not be considered as the "origin" of chaos in food chains, at least for the ranges of parameters which have been considered up to now in simulations (see Table 1).

\subsection{Bifurcation curves rooted at point $M$}

The bifurcation curves in the parameter plane $\left(d_{1}, d_{2}\right)$, which emanate from point $M$, have been continued numerically using LOCBIF, see Figure 3.2. The curves $T_{e}$ and $H_{p}$ are horizontal and vertical straight lines, respectively; the transcritical bifurcation curves $T C_{e}$ and $T C_{c}$ end at the $d_{2}$ - 


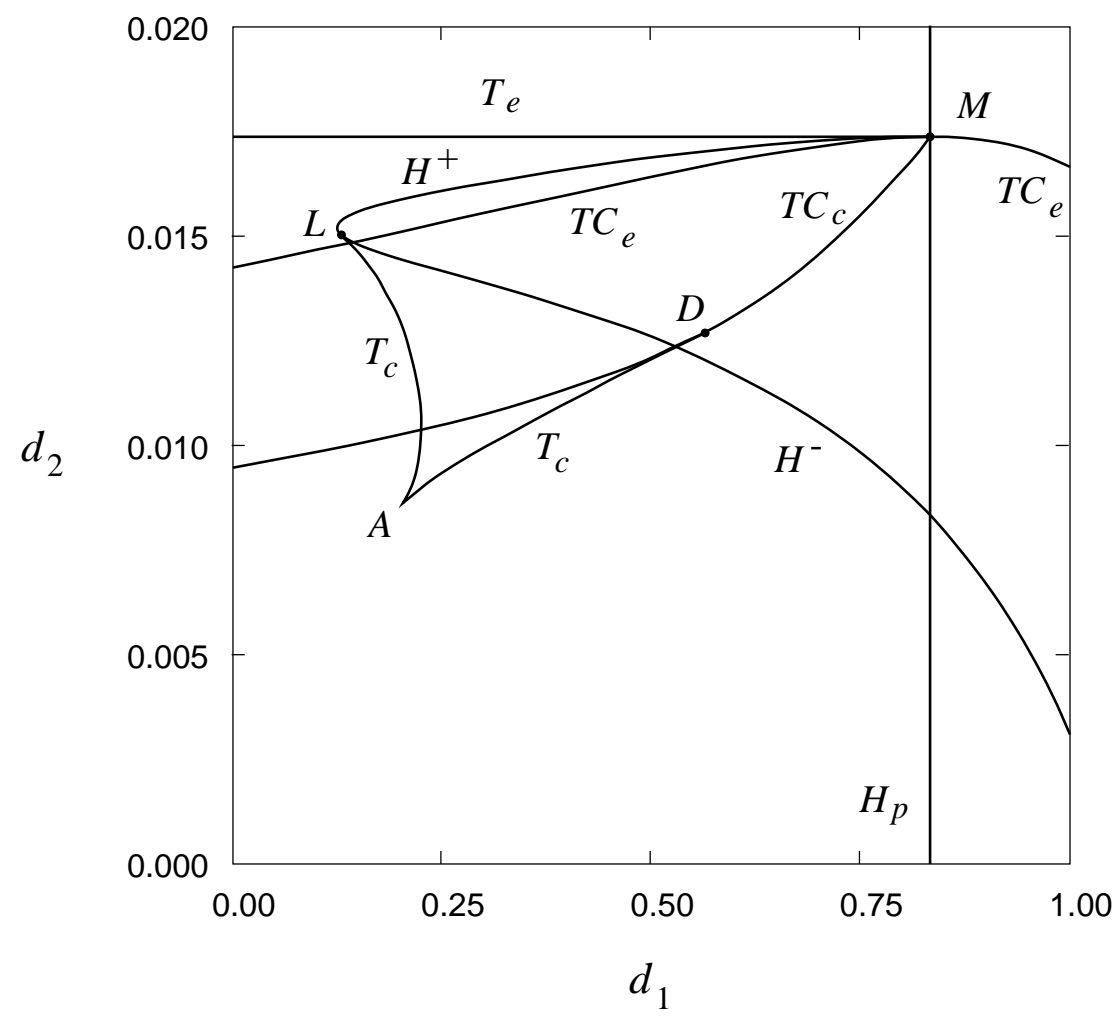

Figure 3.2: Bifurcation curves of system (2.4)-(2.6) originating at the codimension-two point $M$. Parameter values are specified in the text.

axis, while the Hopf bifurcation curve $H$ has a more peculiar form. Its associated first Lyapunov coefficient (1.e., the real part of the cubic coefficient in the normal form) is positive close to $M$ and decreases between $M$ and $L$, where it changes sign. This means that the Hopf bifurcation is subcritical from $M$ to $L$ (on the segment $H^{+}$) and supercritical elsewhere (on the segment $H^{-}$). Therefore (for example, see Arnold [3]), there exists a bifurcation curve $T_{c}$ originating at point $L$ and corresponding to the fold bifurcation (collision) of two limit cycles. Continuation shows that this latter curve has another codimension two singularity, namely a cusp, at point $A$, where three limit cycles collide simultaneously. The curve $T_{c}$ terminates at a point $D$ on the transcritical bifurcation curve $T C_{c}$, where a cycle passes through the invariant plane $x_{3}=0$ : when approaching point $D$ along $T_{c}$, the two colliding cycles "hit" the invariant face. Thus, the curve $T_{c}$ connects the codimension-two bifurcation points $L$ and $D$. We will not list all equilibria and cycles related to the bifurcation curves presented in Figure 3.2, however note that a unique stable limit cycle exists for low values of $d_{1}$ just below curve $T C_{c}$.

\subsection{Cycle bifurcations and chaos}

The bifurcation curves obtained so far form a bifurcation diagram connected with the bifurcation point $M$. However, further numerical analyses show that the actual bifurcation diagram is much more complex and apparently involves an infinity of limit cycle bifurcation curves that are disconnected from the above ones. Figure 3.3 shows some of the bifurcation curves relating to limit cycles. The continuous curves denoted by $F_{k}$ are period doubling (flip) bifurcation curves of cycles, while the dotted curves labeled by $T_{k}$ refer to fold (tangent) bifurcations of cycles. The continuation with respect to $d_{1}$ of the stable limit cycle existing below curve $T C_{c}$ near the vertical axis (see Figure 3.2 ) reveals a cascade of period doubling bifurcations as $d_{1}$ increases. An interesting feature of the flip bifurcation curves is that they are properly ordered, giving rise to a Feigenbaum-like cascade, for 


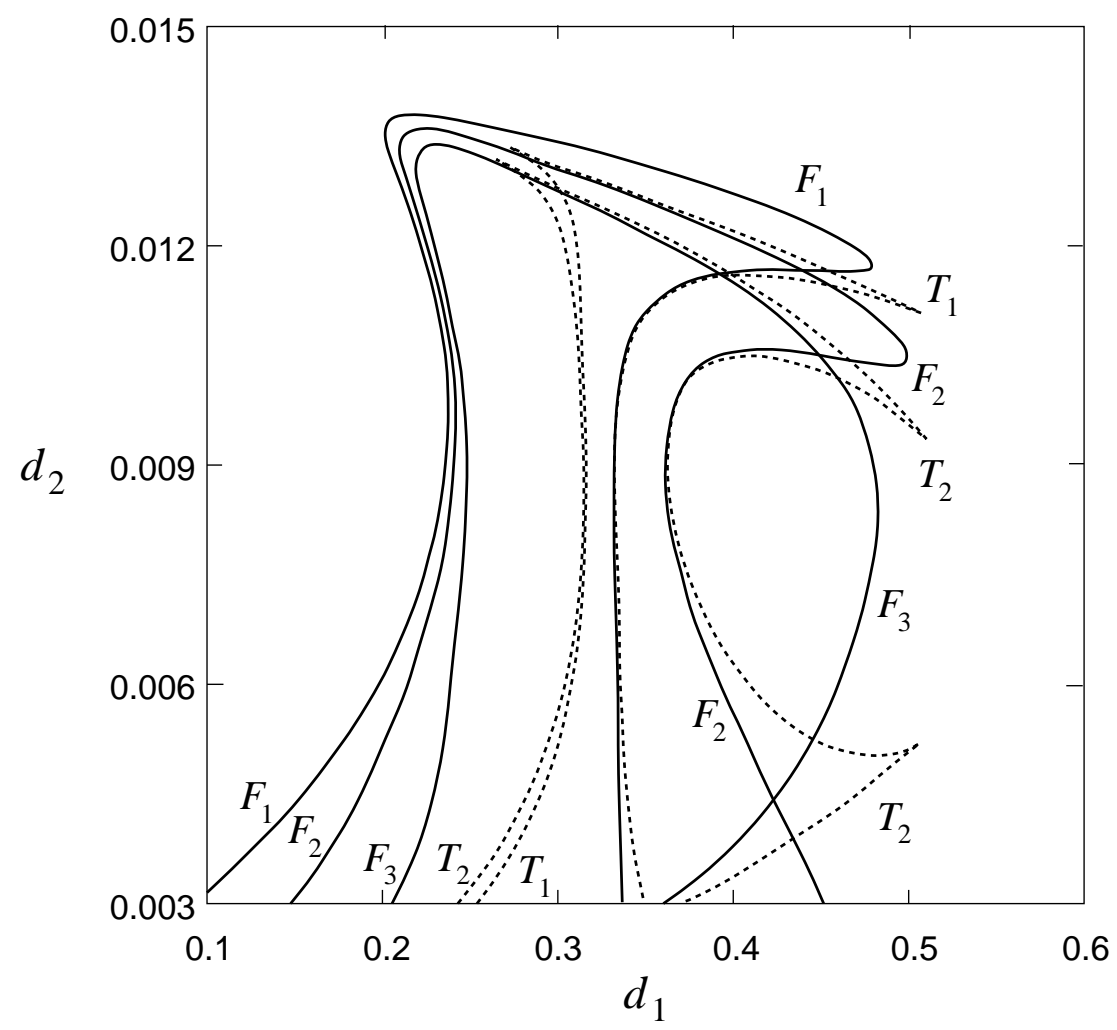

Figure 3.3: Bifurcation curves corresponding to limit cycle bifurcations.

$d_{1}<0.25$ but intersect for $d_{1}>0.25$.

In order to explain these intersections, one can consider a cycle manifold of the system, i.e. a two-dimensional manifold in the $\left(x_{1}, d_{1}, d_{2}\right)$-space specified by the $x_{1}$-coordinates of the cycles in a cross-section $x_{2}=$ const. Notice that for the same value $\left(d_{1}, d_{2}\right)$ of the parameters, $x_{1}$ can have different values since multiple cycles can exist: in other words the cycle manifold can be folded. Consider now Figure 3.4, in which the region containing the intersection point of the flip curves $F_{1}$ and $F_{2}$ is magnified. The projection onto the $\left(d_{1}, d_{2}\right)$-parameter plane of the cycle manifold has a cusp singularity at point $A_{1}$, where two fold branches $T_{1}^{(1)}$ and $T_{1}^{(2)}$ meet tangentially, forming a "horn". Nearby this point, the flip bifurcation curves $F_{1}$ and $F_{2}$ lie on two different sheets of the manifold, so that their projections onto the $\left(d_{1}, d_{2}\right)$-plane can intersect at the point $B_{1}$. Similar horns are associated to each intersection point of any pair of flip curves. Moreover, some of these horns are connected. Figure 3.4 shows, for example, that the fold bifurcation curve $T_{1}^{(2)}$ ends at a second cusp point, namely $A_{2}$, thus forming a second horn with the fold curve $T_{1}^{(3)}$ (which practically coincides with $F_{4}$ ). This second horn is obviously associated to the nearby intersection point of the flip bifurcation curves $F_{2}$ and $F_{4}$.

In conclusion, putting together Figure 3.2 and Figure 3.3, one obtains the diagram depicted in Figure 3.5. This diagram indicates that the chaotic region is bounded in a complicated manner by flip and fold bifurcation curves that have little to do, if anything at all, with point $M$. Entering the chaotic region from the left (by increasing $d_{1}$ ) implies a straightforward cascade of supercritical period doublings. In other words, a high-frequency cycle, due to prey-predator interaction, like the one existing close to the $d_{2}$-axis below $T C_{c}$, gradually becomes a low-frequency cycle. On the contrary, entering the chaotic region from the right (by decreasing $d_{1}$ ) might involve a much more complex sequence of events, because frequency switches (i.e. catastrophic transitions between stable cycles with different periods) are associated with the crossing of each horn. 


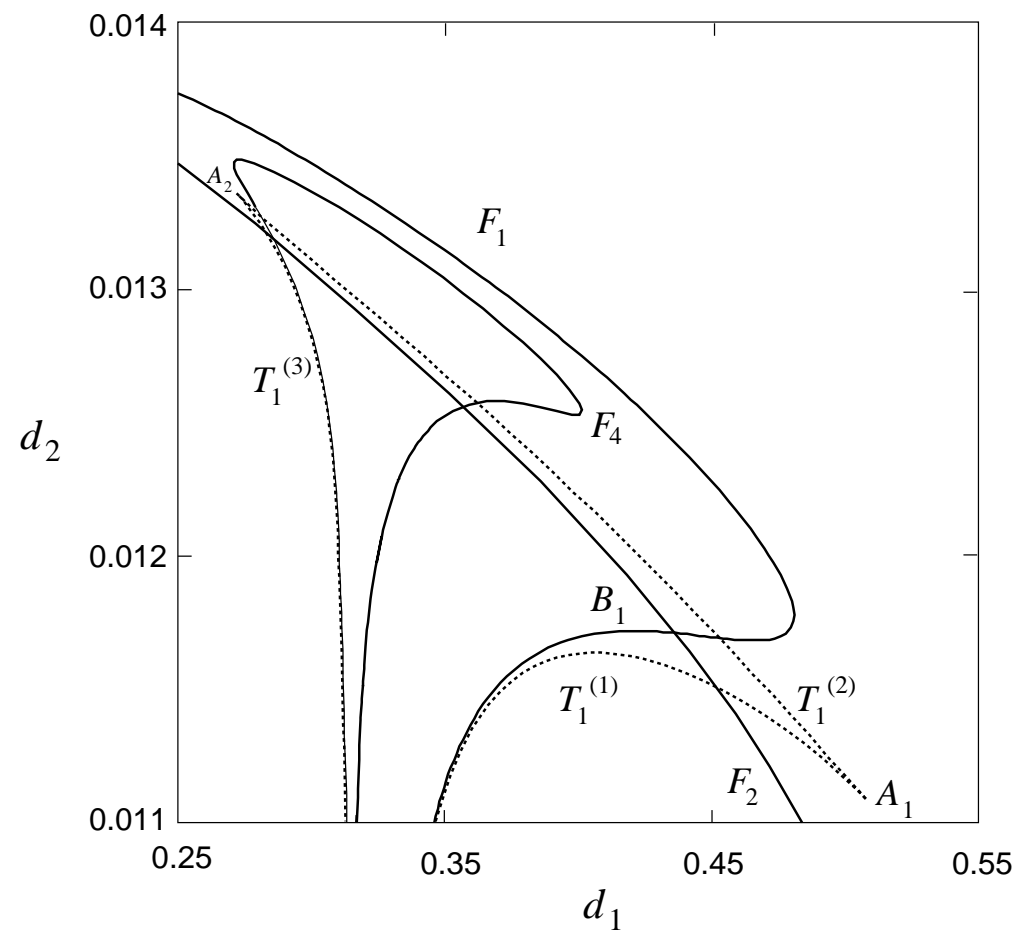

Figure 3.4: Details of the bifurcation diagram.

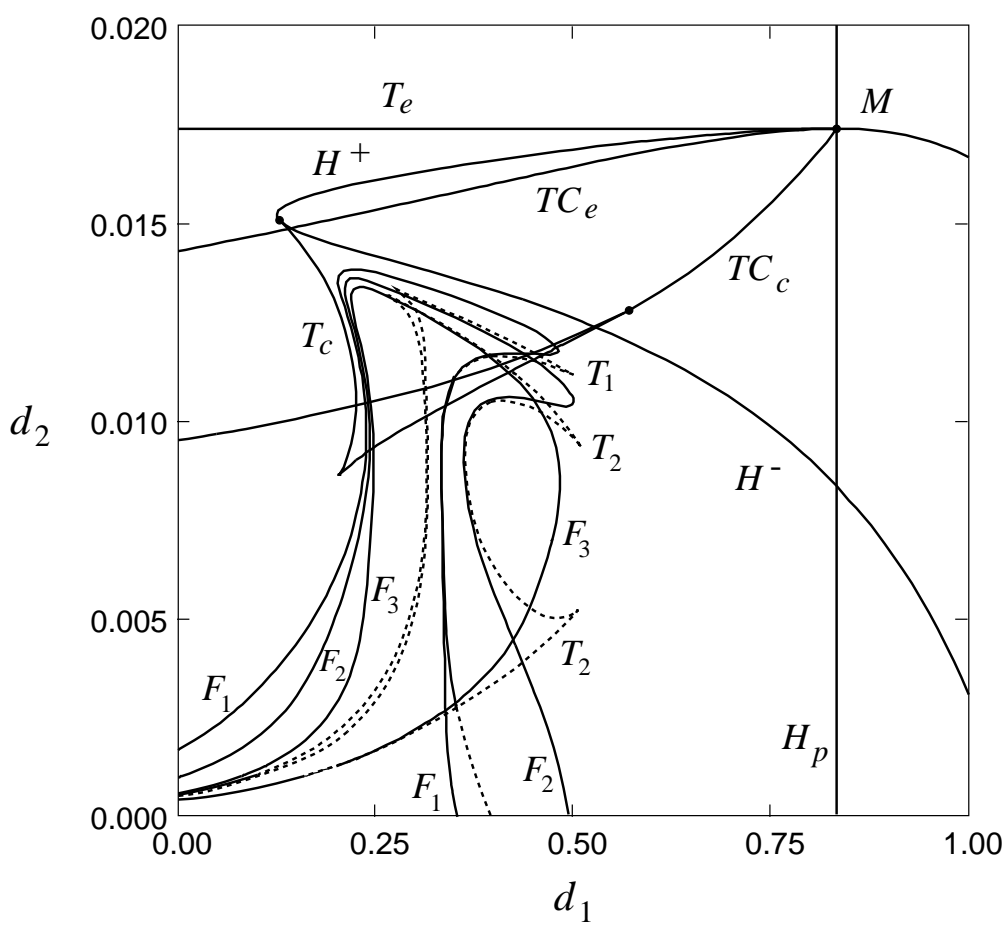

Figure 3.5: Bifurcation diagram of system (2.4) - (2.6). Strange attractors can be found in a vertical band confined between $d_{1} \cong 0.2$ and $d_{1} \cong 0.4$. 


\section{SINGULAR-PERTURBATION ANALYSIS}

The bifurcation diagram obtained in the previous section shows that system (2.4)-(2.6) behaves chaotically in a vertical band confined between $d_{1} \cong 0.2$ and $d_{1} \cong 0.4$ (see Figure 3.5). This feature of the diagram can be explained, at least to a certain extent, by means of singular-perturbation analysis, assuming that the superpredator has slow dynamics relative to prey and predator. This is, actually, the case in all simulation studies referred to in Table 1, where the parameters $a_{2}$ and $d_{2}$ of the superpredator are smaller than the corresponding parameters $a_{1}$ and $d_{1}$ of the predator. The analysis shows that the strange attractors of the system (2.4)-(2.6) have the form of a "tea-cup", and that the domain in the $\left(d_{1}, d_{2}\right)$-plane where strange attractors can be found is specified by some bifurcations of the reduced order system (2.4)-(2.5) with constant superpredator $x_{3}$.

Deliberately leaving out all the technical details which support the singular perturbation approach (the interested reader can refer to Muratori and Rinaldi [27], where the standard food chain is studied), the analysis is carried out in two steps, described in the following two subsections. In the first step, all the stable equilibria and limit cycles of the fast (prey-predator) subsystem (2.4)-(2.5) are determined for all constant values of $x_{3}$. In the second step, the equilibrium manifold of the slow equation (2.6) is introduced and used to derive "singular" orbits that approximate the real orbits of the system.

\subsection{Bifurcation diagram of the fast subsystem}

The second-order "fast" prey-predator system

$$
\begin{aligned}
& \frac{d x_{1}}{d t}=x_{1}\left(1-x_{1}-\frac{a_{1} x_{2}}{1+b_{1} x_{1}}\right), \\
& \frac{d x_{2}}{d t}=x_{2}\left(\frac{a_{1} x_{1}}{1+b_{1} x_{1}}-d_{1}-\frac{a_{2} x_{3}}{1+b_{2} x_{2}}\right),
\end{aligned}
$$

where $x_{3}$ is a parameter, is now considered. This system is of interest per se, because in many natural food chains the superpredator has alternative sources of food and is, therefore, roughly constant. The dynamic behavior of (4.1)-(4.2) is already well-understood. Its Hopf, fold and transcritical bifurcations have been analyzed by Muratori and Rinaldi [24, 25], while homoclinic bifurcations have been detected through simulations by Scheffer [32], and then studied analytically and numerically by Kuznetsov et al. [19], where a complete two-parameter bifurcation diagram has been obtained.

Figure 4.1 presents the bifurcation diagram of (4.1)-(4.2) in the parameter plane $\left(d_{1}, x_{3}\right)$ together with generic phase portraits. The diagram has been computed with all parameters fixed at the reference values used in the preceding analysis. The parameter $d_{1}$ has been selected as control parameter because our target is the interpretation of the bifurcations of (2.4)-(2.6) with respect to $d_{1}$ and $d_{2}$. Reading this bifurcation diagram is quite simple. The equilibrium $(1,0)$ of $(4.1)-(4.2)$ undergoes a transcritical bifurcation along the line $T C$. Point $D$ on this line is a codimension-two bifurcation point corresponding to a degenerate transcritical bifurcation. This point is, therefore, the root of a fold bifurcation curve $T$, along which two strictly positive equilibria (a saddle and a node) appear. Also the fold curve $T$ contains a codimension-two bifurcation point, namely a Bogdanov-Takens point $B T$, at which system (4.1)-(4.2) has an equilibrium with a double zero eigenvalue. Point $B T$ is located outside the positive quadrant of the $\left(d_{1}, x_{3}\right)$-plane but, nevertheless, plays an important role, since a Hopf bifurcation curve $H$ and a homoclinic bifurcation curve $P$ originate at this point [3] and then enter the positive quadrant. Notice that the Hopf curve $H$ intersects the transcritical line $T C$ at $d_{1} \cong 0.46$, while the homoclinic curve $P$ has a turning point at $d_{1} \cong 0.165$.

For each value of $d_{1}$, the invariant sets (equilibria and cycles) of (4.1)-(4.2) can be drawn in the $\left(x_{1}, x_{2}, x_{3}\right)$-space. In such a representation, an equilibrium appears as a curve, while a cycle gives rise to a paraboloid-like surface with the vertex corresponding to a Hopf bifurcation point. The invariant sets of (4.1)-(4.2) are shown in Figure 4.2 for three values of $d_{1}$ corresponding to the vertical lines (a),(b) and (c) in Figure 4.1. The bifurcation sequences appearing along these lines are different and along line (c) they are five, instead of three, because of two extra bifurcations, namely a "direct" and a "reverse" homoclinic bifurcation. The appearance of these two homoclinic bifurcations leads to the cutting of the paraboloid-like surface into two pieces. 

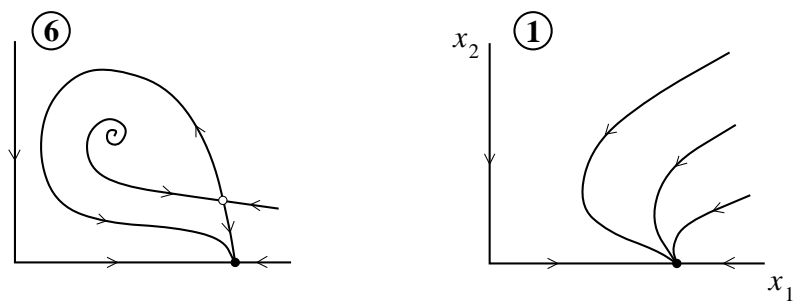

(c) (b)

(a)
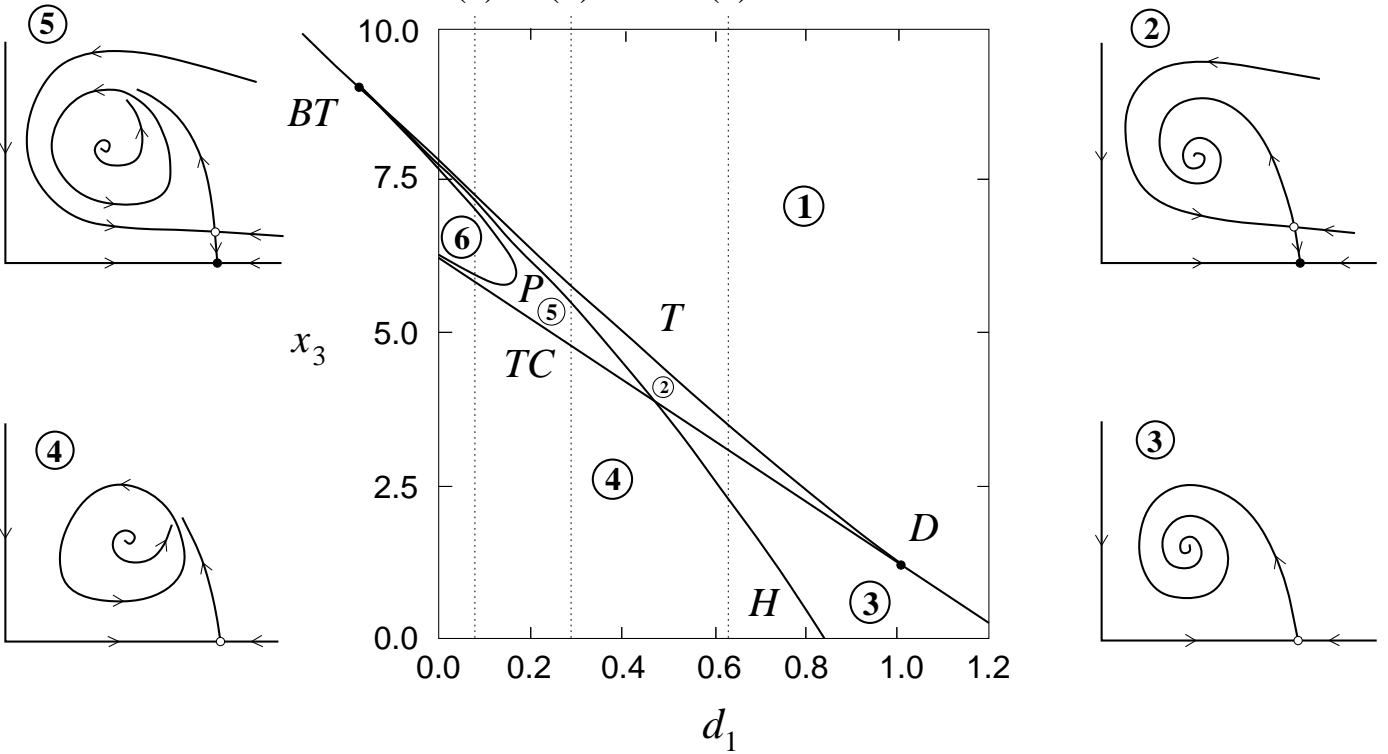

(4)
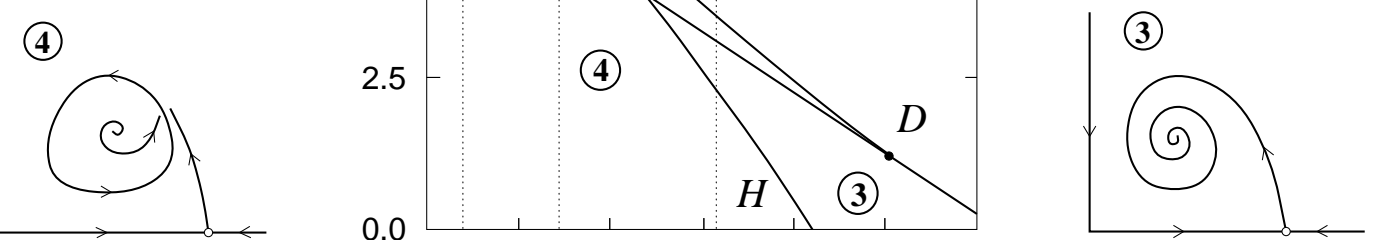

Figure 4.1: Parametric and typical phase portraits of the fast system (4.1)-(4.2) for the reference values of the parameters.

4.2 Creation and cutting of a "tea-cup" attractor

The nontrivial equilibrium manifold of the slow (superpredator) equation

$$
\frac{d x_{3}}{d t}=x_{3}\left(\frac{a_{2} x_{2}}{1+b_{2} x_{2}}-d_{2}\right)
$$

is the plane given by

$$
x_{2}=\frac{d_{2}}{a_{2}-b_{2} d_{2}},
$$

which approaches the coordinate plane $\left(x_{1}, x_{3}\right)$ as $d_{2} \rightarrow 0$. Above this plane, the superpredator increases $\left(\dot{x}_{3}>0\right)$, so that orbits move to the right, while below, orbits move to the left $\left(\dot{x}_{3}<0\right)$. Moreover, if $d_{2}$ is sufficiently small, the plane lies entirely below both the paraboloid-like surface and the segment $H T$ of the stable equilibrium coming out from its vertex (see Figure 4.2). This is assumed in the following discussion.

Singular-perturbation analysis, then, says that the orbits of system (4.1)-(4.3) are approximated by so called "singular" orbits, which are composed of slow motions along the stable equilibrium (or cycle) manifolds of the fast subsystem in the direction dictated by the sign of $\dot{x}_{3}$, and fast jumps near catastrophic bifurcation points of the fast subsystem. Some of the delicate mathematical problems arising in this context, including "canard solutions", are dealt with by Muratori and Rinaldi [27] and Terman [35].

In the case presented in Figure 4.2(a), the configuration of the invariant manifolds implies the existence of a singular limit cycle composed of four concatenated segments. An initial slow motion 


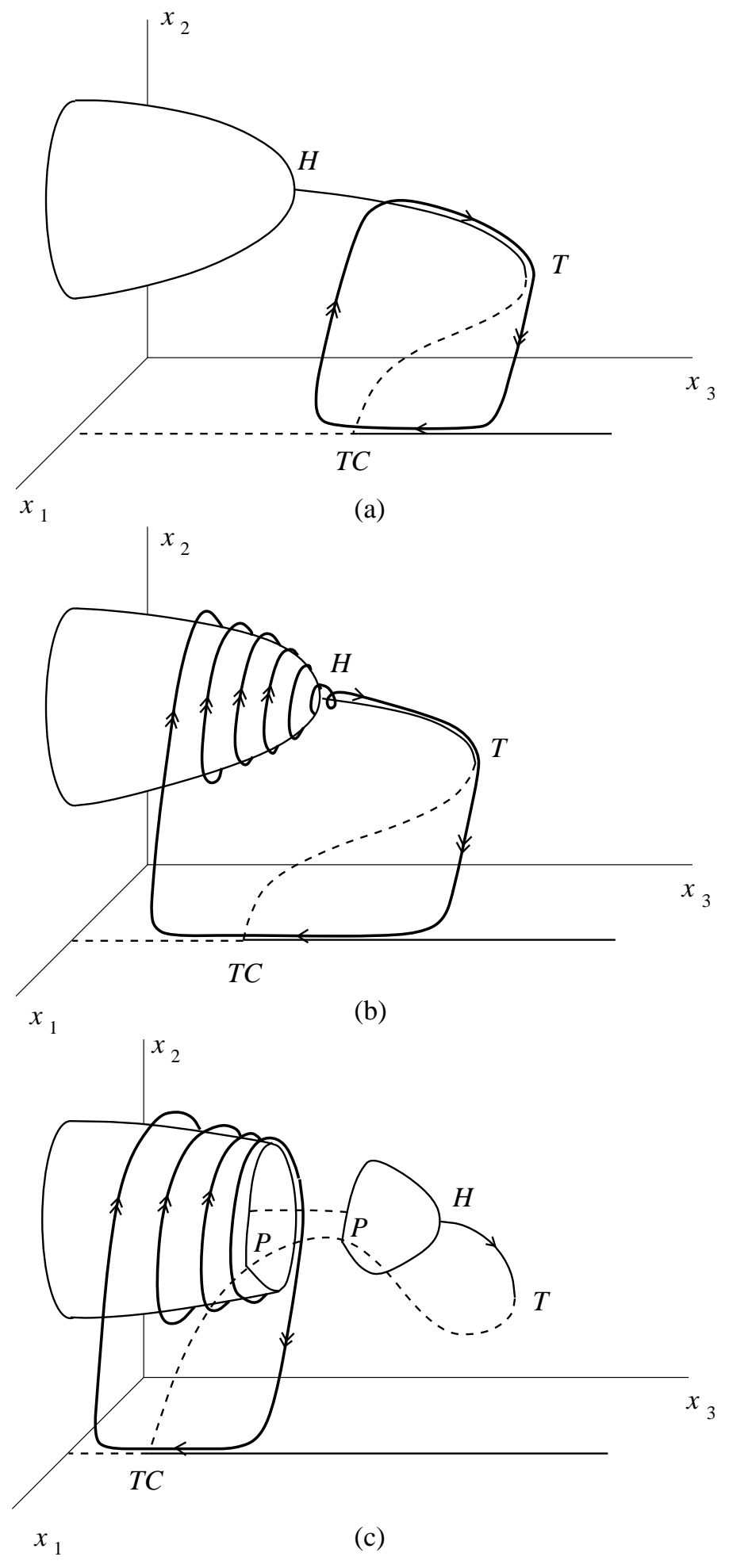

Figure 4.2: Equilibria and cycles of the fast system (4.1)-(4.2) plotted against $x_{3}$ for the values of $d_{1}$ corresponding to lines (a),(b) and (c) in Figure 7. The bold curves indicate orbits of (2.4)-(2.6). Double (single) arrows indicate fast (slow) motion. 
along the equilibrium manifold $H T$ is followed by a fast jump from the bifurcation point $T$ to the trivial equilibrium on the $\left(x_{1}, x_{3}\right)$-plane. During this fast transition, the orbit crosses the manifold $\dot{x}_{3}=0$ so that the third segment of the singular cycle develops from right to left along the line of trivial equilibria. For technical reasons described in [27], the slow motion continues beyond the transcritical point $T C$ and then jumps back to the branch $H T$, thus closing the cycle. In Figure $4.2(\mathrm{a})$, the real cycle of a slow-fast food chain is sketched and its fast parts are indicated with double arrows.

In the case depicted in Figure 4.2(b), the resulting singular cycle is more complex. It has the peculiar form of a "tea-cup" with a long handle. The real motion in the slow-fast system can be either cyclic or chaotic. In some of the papers devoted to simulations of food chains, there are figures showing this latter type of attractor.

Finally, in the case of Figure 4.2(c), which was not studied in [27], the "tea-cup" attractor is "shorter". The comparison of Figure 4.2(b) and Figure 4.2(c) shows that the loss of the bottom of the cup is due to the appearance of a double homoclinic orbit in the fast system (4.1),(4.2). If the gap between the two emerging homoclinic bifurcations is wide enough, all orbits starting near the right piece of the paraboloid eventually reach the left one and then never visit the right piece any more. The motion near the left part of the "tea-cup" can also be chaotic or can degenerate into a high-frequency predator-prey cycle (see Figure 1.1(a)).

The described sequence of figures corresponds to decreasing values of $d_{1}$ (see the vertical lines (a),(b) and (c) in Figure 4.1). Thus, Figure 4.2 implies that, for decreasing values of $d_{1}$, the attractor of the system undergoes significant transformations: from a simple low-frequency cycle, to a "tea-cup" attractor and, finally, through a "cut tea-cup" attractor, to a high-frequency cycle. The "tea-cup" attractor appears at a value of $d_{1}$ corresponding to the intersection of the Hopf and the transcritical curves, $H$ and $T C$, i.e. for $d_{1} \cong 0.46$. The cutting takes place at the value of $d_{1}$ which corresponds to the turning point of the homoclinic bifurcation curve $P$ in the $\left(d_{1}, x_{3}\right)$-plane (see Figure 4.1 ). As we have noticed above, for the reference parameter setting this value of $d_{1}$ is approximately equal to 0.165. Notice that the chaotic band of Figure 3.5 is located between these two critical values of $d_{1}$.

\section{DISCUSSION AND CONCLUDING REMARKS}

A bifurcation diagram of the standard food chain model (logistic prey and Holling type II predator and superpredator) has been produced and discussed in this paper with the aim of classifying the main modes of behavior of food chains. The bifurcations have been determined by studying the normal form of a codimension-two point and by extending the bifurcation curves emerging from that point by means of a continuation technique. The analysis is more systematic than those recently published by Klebanoff and Hastings [17] and McCann and Yodzis [22] and confirms some of their results and conjectures. The normal form derived in this paper is parameter-dependent and takes into account the specific functional form of the model. This simplifies the analysis because it reduces to two the number of possible cases (identified by the sign of a strategic parameter of the normal form). Actually, only one of these two cases is analysed in the paper because all the known studies on the standard food chain (see Table 1) fall into the same class.

The bifurcation diagram (see Figure 3.5) indicates that strange attractors are present in a region of the parameter space which is far from the codimension-two point, thus proving that the existence of chaos has nothing to do with this point. This is confirmed by a singular-perturbation analysis in which the superpredator is assumed to be the slow component of the system, while the prey-predator subsystem is the fast component. This is very often the case in natural food chains, algae-zooplanktonfish being a typical example. A purely geometric approach showed that a simple low-frequency cycle, as sketched in Figure 1.1(b), can turn into a "tea-cup" attractor (see Figure 1.1(c)) under variation of some parameter. This happens when a Hopf bifurcation in the second-order fast subsystem coincides with a transcritical bifurcation. Under further parameter variation, this attractor can split into two pieces. This event is associated with the appearance of a double homoclinic orbit in the fast subsystem. The "cut tea-cup" attractor finally becomes a simple high-frequency cycle, as sketched in Figure 1.1(a). The numerical analysis carried out on this problem clearly suggests that the appearance and cutting 
of the tea-cup are the indicators of chaos. Further work is needed on this subject, since what is known on third-order slow-fast systems is not enough to tackle the problem. In particular, it would be very interesting to know if the existence of the tea-cup is related with the presence of Shil'nikov's homoclinic orbits in the model. Indeed, examples of such kind of orbits (not shown in the paper) have been obtained numerically (see also [22]).

Many curves appearing in Figure 3.5 refer to bifurcations involving only unstable equilibria and/or unstable cycles. Of course, these bifurcation curves are needed to fully understand the transformations and the matching of all attracting, repelling and saddle invariant sets. But, if the final target of the analysis is the classification of the stable modes of behavior, these bifurcation curves are of no help and can, therefore, be erased from the final diagram, which then becomes much more simple. Figure

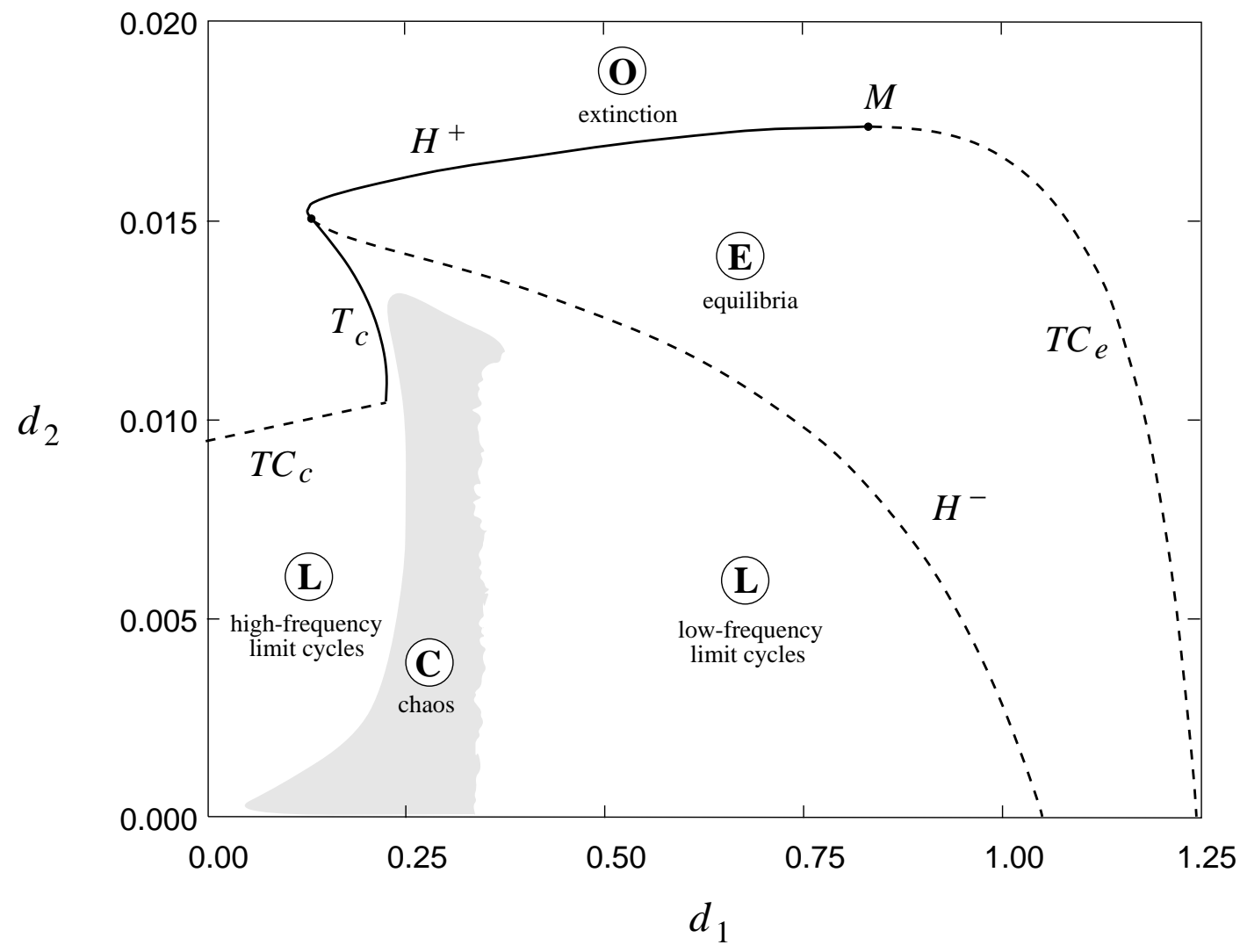

Figure 5.1: Reduced bifurcation diagram of system (2.4)-(2.6) identifying regions of coexistence of populations: $\mathbf{E}$ steady coexistence; $\mathbf{L}$ cyclic coexistence; $\mathbf{C}$ chaotic coexistence.

5.1 shows a reduced diagram obtained from Figure 3.5 (recall that $d_{1}$ and $d_{2}$ are the rescaled death rates of predator and superpredator). Food chains corresponding to points in region $\mathbf{O}$ of this diagram are characterized by the extinction of the superpredator, while in all other regions stable coexistence of the three populations is possible. More precisely, in region $\mathbf{E}$, only steady coexistence is possible because the unique positive attractor existing in that region is an equilibrium. Similarly, in region $\mathbf{L}$ coexistence is possible only on a limit cycle, while in region $\mathbf{C}$ stable chaotic behavior is also present. Nevertheless, in some parts of these regions, coexistence is not guaranteed for all initial conditions. This happens when there exists also a trivial attractor involving the extinction of the superpredator. For example, in the left upper part of region $\mathbf{E}$ (actually, above curve $T C_{c}$ of Figure 3.5), the system has also a stable prey-predator limit cycle and, indeed, in that region the fate of the superpredator depends upon the initial population densities. Moreover, in region $\mathbf{L}$ there are also subregions, where there are two (or more) stable positive limit cycles. 
The multiplicity of the attractors is certainly one of the most interesting features of food chains. It explains, for example, why after an exogenous shock (like a forest fire, a flood or an epidemic) a food chain might radically change its mode of behavior. In fact, a shock can suddenly transfer the state of the system from its usual basin of attraction into another basin of attraction, so that, after a transient, the system will behave in a different way. The multiplicity of the attractors can also explain why small perturbations of the environment (like temperature or $p H$ variations in a lake) can entail macroscopic consequences on the populations (like frequency switches in the cyclic regime of the food chain or extinction of the top predator). This happens when the parameter perturbation is interpretable as the crossing of one of the catastrophic bifurcation curves (indicated with continuous lines in Figure 5.1). Thus, for example, a slow but continuous increase of the rate of harvest of the superpredator (increase of $d_{2}$ ) will inevitably end up with the extinction of such population, but in two substantially different ways: with a clear evidence of decay, in food chains with very high or very low death rate $d_{1}$, and with almost no warning, for intermediate values of $d_{1}$.

Figure 5.1 also shows where the food chain can be chaotic (region $\mathbf{C}$ ). This region is a rather narrow vertical band in the parameter space $\left(d_{1}, d_{2}\right)$. Its left boundary is very regular and corresponds to the limit of a sequence of flip bifurcation curves. In contrast, the right boundary is more complex, because the flip bifurcation curves (see Figure 3.5) intersect, giving rise to infinitely many "horns". Near the edge of each of them there are two stable cycles. Going into the limit, these horns become infinitely thin and form the right boundary of the chaotic region. Thus, a chaotic food chain should be stabilizable either by decreasing or by increasing $d_{1}$ (for example, by varying the rate of harvest of the predator). In the first case ( $d_{1}$ decreasing), one would emerge from chaos through a reverse cascade of period doublings, thus tending towards a high-frequency prey-predator cycle, as predicted by the singular-perturbation analysis. In the second case ( $d_{1}$ increasing), one would emerge from chaos passing through the above described horns, each one implying a catastrophic transition. In conclusion, while the left route to chaos is a classical cascade of period doublings with no catastrophic transitions, the right route to chaos is much more spectacular and implies a cascade of catastrophic transitions.

\section{Acknowledgement}

This work has been partially supported by the International Institute for Applied Systems Analysis (IIASA), Laxenburg, Austria, and by the Italian Ministry of Scientific Reserach and Technology, contract MURST 40\% Teoria dei sistemi e del controllo.

\section{APPENDIX: NORMAL FORM DERIVATION}

This Appendix is devoted to the derivation of the parameter-dependent normal form (3.1) for the codimension-two point at which system (2.4)-(2.6) has an equilibrium $x^{0}$ on the face $x_{3}=0$ with associated eigenvalues

$$
\lambda_{1}=0, \lambda_{2,3}= \pm i \omega_{0}, \omega_{0}>0 .
$$

The invariant technique based on adjoint eigenvectors (see [18]) is used.

\subsection{Preliminary linear transformations}

Write system (2.4)-(2.6) for $d$ close to $d^{*}$ near the equilibrium $x^{0}$ in the new coordinates $y=x-x^{0}$, thus obtaining

$$
\dot{y}=J y+\Psi(y), \quad y=\left(y_{1}, y_{2}, y_{3}\right)^{T} \in \mathbb{R}^{3},
$$

where $J$ is the Jacobian matrix of (2.4)-(2.6) evaluated at $x^{0}$ (the dependence upon the parameters $d=\left(d_{1}, d_{2}\right)$ is not shown for simplicity). At $d=d^{*}$, one has

$$
J^{*}=\left(\begin{array}{ccc}
0 & -\frac{a_{1}\left(b_{1}-1\right)}{b_{1}\left(b_{1}+1\right)} & 0 \\
\frac{1}{b_{1}} & 0 & -\frac{a_{2}\left(b_{1}+1\right)^{2}}{b_{2}\left(b_{1}+1\right)^{2}+4 a_{1} b_{1}} \\
0 & 0 & 0
\end{array}\right) .
$$


The function $\Psi(y)$ has a Taylor expansion starting with quadratic terms that can be written as

$$
\Psi(y)=\left(\begin{array}{c}
\psi_{1}\left(y_{1}, y_{2}\right) \\
\psi_{2}\left(y_{1}, y_{2}, y_{3}\right) \\
y_{3} \varphi\left(y_{2}\right)
\end{array}\right),
$$

where the form of the last component reflects the invariance of the face $\left(x_{1}, x_{2}\right)$ in model (2.4)-(2.6), and $\varphi=\Gamma y_{2}+O\left(y_{2}^{2}\right)$, with the critical value at $d=d^{*}$

$$
\Gamma^{*}=\frac{16 a_{1}^{2} a_{2} b_{1}^{2}}{\left[b_{2}\left(b_{1}+1\right)^{2}+4 a_{1} b_{1}\right]^{2}}>0 .
$$

The Jacobian matrix $J$ has a real eigenvalue

$$
\lambda_{1}=\beta(d)=\frac{b_{1} d_{1}\left(a_{1}-b_{1} d_{1}-d_{1}\right)-a_{1} d_{1}}{2 a_{1}\left(a_{1}-b_{1} d_{1}\right)},
$$

and a pair of complex-conjugate eigenvalues $\lambda_{2}=\lambda, \lambda_{3}=\bar{\lambda}$, where

$$
\lambda(d)=\alpha(d)+i \omega(d)
$$

with

$$
\alpha(d)=\frac{\left(a_{1}-b_{1} d_{1}-d_{1}\right)\left(a_{2}-b_{2} d_{2}\right)-\left(a_{1}-b_{1} d_{1}\right)^{2} d_{2}}{\left(a_{1}-b_{1} d_{1}\right)^{2}+b_{2}\left(a_{1}-b_{1} d_{1}-d_{1}\right)},
$$

and

$$
\omega\left(d^{*}\right)=\omega_{0}=\frac{1}{b_{1}} \sqrt{\frac{a_{1}\left(b_{1}-1\right)}{b_{1}+1}}>0 .
$$

Obviously, $\beta\left(d^{*}\right)=\alpha\left(d^{*}\right)=0$. Moreover, a real eigenvector $q$ is associated to $\lambda_{1}$, and a complex eigenvector $w$ is associated to $\lambda$,

$$
J q=\beta q, J w=\lambda w .
$$

Thus, any real vector $y$ can be decomposed, for all parameter values $d$ close to $d^{*}$, as

$$
y=\xi q+z w+\bar{z} \bar{w},
$$

where $\xi$ is real and $z$ is complex. These new "coordinates" $(\xi, z)$ can be expressed explicitly in terms of the eigenvectors $p$ and $v$ of the transposed Jacobian matrix $J^{T}$ :

$$
J^{T} p=\beta p, J^{T} v=\bar{\lambda} v
$$

Provided the eigenvectors are normalized so that $\langle p, q\rangle=\langle v, w\rangle=1$, where $\langle p, q\rangle=\bar{p}_{1} q_{1}+\bar{p}_{2} q_{2}+\bar{p}_{3} q_{3}$ is the standard scalar product in $\mathbb{C}^{3}$, the following simple formulae are valid:

$$
\xi=\langle p, y\rangle, z=\langle v, y\rangle,
$$

since $\langle p, w\rangle=\langle v, q\rangle=0$. In the coordinates $(\xi, z),(1.1)$ takes the form

$$
\left\{\begin{array}{l}
\dot{\xi}=\beta \xi+G(\xi, z, \bar{z}) \\
\dot{z}=\lambda z+H(\xi, z, \bar{z})
\end{array}\right.
$$


where the functions $G$ and $H$ (depending also on $d$ ) have the following expressions:

$$
G(\xi, z, \bar{z})=\langle p, \Psi(\xi q+z w+\bar{z} \bar{w})\rangle, H(\xi, z, \bar{z})=\langle v, \Psi(\xi q+z w+\bar{z} \bar{w})\rangle
$$

These functions can be developed into Taylor series as

$$
G(\xi, z, \bar{z})=\delta \xi^{2}+g_{110} \xi z+g_{101} \xi \bar{z}+g_{111} \xi|z|^{2}+\frac{1}{2} g_{120} \xi z^{2}+\frac{1}{2} g_{102} \xi \bar{z}^{2}+O\left(\|(\xi, z, \bar{z})\|^{4}\right)
$$

and

$$
H(\xi, z, \bar{z})=\sum_{2 \leq i+j+k \leq 3} \frac{1}{i ! j ! k !} h_{i j k} \xi^{i} z^{j} \bar{z}^{k}+O\left(\|(\xi, z, \bar{z})\|^{4}\right) .
$$

Here all the coefficients $g$ 's and $h$ 's, as well as $\delta$, are functions of the parameter $d$. Note that $\xi$ can be factored out in the function $G$ because of the invariance of the plane $y_{3}=0$. A more important and less obvious property is that the coefficient $\delta$ of the $\xi^{2}$-term vanishes at $d=d^{*}$. Indeed, the third component of the eigenvector $w$ is zero: $w_{3}=0$, while the eigenvector $p$ is orthogonal to the face $y_{3}=0$, so that $p_{1}=p_{2}=0$. Moreover, $p_{3} q_{3}=1$ due to the normalization. Thus,

$$
G=\langle p, \Psi(z w+\bar{z} \bar{w}+\xi q)\rangle=p_{3} q_{3} \xi \varphi\left(z w_{2}+\bar{z} \bar{w}_{2}+\xi q_{2}\right)=\Gamma p_{3} q_{3} q_{2} \xi^{2}+\ldots=\Gamma q_{2} \xi^{2}+\ldots
$$

At $d=d^{*}$, the normalized eigenvectors can be selected in the form

$$
q^{*}=\left(\frac{a_{2}\left(b_{1}+1\right)^{2}}{4 a_{1} b_{1}+b_{2}\left(b_{1}+1\right)^{2}}, 0, \frac{1}{b_{1}}\right)^{T}, p^{*}=\left(0,0, b_{1}\right)^{T},
$$

and

$$
w^{*}=\left(\frac{i b_{1} \omega_{0}}{2}, \frac{1}{2}, 0\right)^{T}, v^{*}=\left(\frac{i}{b_{1} \omega_{0}}, 1,-\frac{i a_{2}\left(b_{1}+1\right)^{2}}{\omega_{0}\left[4 a_{1} b_{1}+b_{2}\left(b_{1}+1\right)^{2}\right]}\right)^{T} .
$$

As one can see, $q_{2}^{*}=0$, so that $\delta=0$ for $d=d^{*}$.

The critical values of the coefficients used in the following are given by the formulae:

$$
\begin{aligned}
g_{110}^{*} & =g_{101}^{*}=\frac{8 a_{1}^{2} a_{2} b_{1}^{2}}{\left[4 a_{1} b_{1}+b_{2}\left(b_{1}+1\right)^{2}\right]^{2}}, \quad g_{111}^{*}=g_{102}^{*}=-\frac{32 a_{1}^{3} a_{2} b_{1}^{3} b_{2}}{\left[4 a_{1} b_{1}+b_{2}\left(b_{1}+1\right)^{2}\right]^{3}}, \\
h_{200}^{*} & =-\frac{2 a_{2}^{2}\left(b_{1}+1\right)^{3}\left[2 \omega_{0} b_{1}-i\left(b_{1}-1\right)\right]}{\omega_{0} b_{1}\left[4 a_{1} b_{1}+b_{2}\left(b_{1}+1\right)^{2}\right]^{2}}, \\
h_{011}^{*}= & -\frac{\omega_{0} b_{1}\left[2 \omega_{0} b_{1}-i\left(b_{1}-1\right)\right]}{2\left(b_{1}+1\right)}, \\
h_{020}^{*}= & \frac{2 \omega_{0}^{2} b_{1}^{2}\left(b_{1}+1\right)-4 a_{1}-i \omega_{0} b_{1}\left(b_{1}^{2}-1-4 a_{1}\right)}{2\left(b_{1}+1\right)^{2}}, \\
\operatorname{Re} h_{110} & =\frac{2 a_{1} a_{2} b_{2}\left(b_{1}+1\right)^{2}}{\left[4 a_{1} b_{1}+b_{2}\left(b_{1}+1\right)^{2}\right]^{2}}-\frac{a_{2}\left(b_{1}^{2}-1\right)}{4 a_{1} b_{1}+b_{2}\left(b_{1}+1\right)^{2}} \\
\operatorname{Re} h_{021} & =\frac{\omega_{0}^{2} b_{1}^{3}\left[3\left(b_{1}+1\right)+2 a_{1}\right]}{\left(b_{1}+1\right)^{3}}, \\
\operatorname{Re} h_{210} & =\frac{4 a_{2}^{2} b_{1}\left(b_{1}+1\right)\left[3\left(b_{1}+1\right)+2 a_{1}\right]}{\left[4 a_{1} b_{1}+b_{2}\left(b_{1}+1\right)^{2}\right]^{2}} .
\end{aligned}
$$




\subsection{Normalization}

Normalization starts by applying a quadratic transformation of variables to (1.4),

$$
\left\{\begin{array}{l}
\eta=\xi+V_{110} \xi z+V_{101} \xi \bar{z} \\
u=z+\frac{1}{2} W_{200} \xi^{2}+\frac{1}{2} W_{020} z^{2}+\frac{1}{2} W_{002} \bar{z}^{2}+W_{101} \xi \bar{z}+W_{011} z \bar{z}
\end{array}\right.
$$

where $\eta$ is real and $u$ is complex, and $V_{i j k}$ and $W_{i j k}$ are suitable coefficients.

The resulting equations can be simplified to

$$
\left\{\begin{array}{l}
\dot{\eta}=\beta \eta+\delta \eta^{2}+\sum_{i+j+k=3} \frac{1}{i ! j ! k !} G_{i j k} \eta^{i} u^{j} \bar{u}^{k}+O\left(\|(\eta, u, \bar{u})\|^{4}\right) \\
\dot{u}=(\alpha+i \omega) u+h_{110} \eta u+\sum_{i+j+k=3} \frac{1}{i ! j ! k !} H_{i j k} \eta^{i} u^{j} \bar{u}^{k}+O\left(\|(\eta, u, \bar{u})\|^{4}\right)
\end{array}\right.
$$

by setting

$$
\begin{gathered}
V_{110}=\frac{g_{110}}{\bar{\lambda}}, V_{101}=\frac{g_{101}}{\lambda}, W_{200}=\frac{h_{200}}{\lambda}, W_{020}=\frac{h_{020}}{\bar{\lambda}}, \\
W_{101}=\frac{h_{101}}{2 \lambda}, W_{002}=\frac{h_{002}}{3 \lambda}, W_{011}=\frac{h_{011}}{\lambda} .
\end{gathered}
$$

Of couse, the above transformation modifies the coefficients of the cubic terms. The cubic coefficients which are needed in the following are given $\left(\right.$ at $\left.d=d^{*}\right)$ by the formulae (see [18]):

$$
\begin{aligned}
G_{300}^{*} & =-\frac{6}{\omega_{0}} \operatorname{Im}\left(g_{110}^{*} h_{200}^{*}\right), \\
G_{111}^{*} & =g_{111}^{*}-\frac{2}{\omega_{0}} \operatorname{Im}\left(g_{110}^{*} h_{011}^{*}\right), \\
H_{210}^{*} & =h_{210}^{*}+\frac{i}{\omega_{0}}\left[h_{200}^{*}\left(h_{020}^{*}-2 g_{110}^{*}\right)-\left|h_{101}^{*}\right|^{2}-h_{011}^{*} \bar{h}_{200}^{*}\right], \\
H_{021}^{*} & =h_{021}^{*}+\frac{i}{\omega_{0}}\left[h_{011}^{*} h_{020}^{*}-2\left|h_{011}^{*}\right|^{2}-\frac{1}{3}\left|h_{002}^{*}\right|^{2}\right] .
\end{aligned}
$$

By performing a transformation involving cubic terms, system (1.6) can be reduced to the following form (where the same notation as in (1.6) is used):

$$
\left\{\begin{array}{l}
\dot{\eta}=\beta \eta+\delta \eta^{2}+\frac{1}{6} G_{300} \eta^{3}+G_{111} \eta|u|^{2}+O\left(\|(\eta, u, \bar{u})\|^{4}\right) \\
\dot{u}=(\alpha+i \omega) u+h_{110} \eta u+\frac{1}{2} H_{210} \eta^{2} u+\frac{1}{2} H_{021} u|u|^{2}+O\left(\|(\eta, u, \bar{u})\|^{4}\right) .
\end{array}\right.
$$

If the complex variable $u$ is written in the exponential form $u=\rho e^{i \theta}$, the above normal form reduces to that given in [17]:

$$
\left\{\begin{array}{l}
\dot{\eta}=\beta \eta+\delta \eta^{2}+A \rho^{2} \eta+B \eta^{3}+O\left(\|(\eta, \rho)\|^{4}\right) \\
\dot{\rho}=\alpha \rho+C \eta \rho+D \eta^{2} \rho+E \rho^{3}+O\left(\|(\eta, \rho)\|^{4}\right) \\
\dot{\theta}=\omega+O(\|(\eta, \rho)\|)
\end{array}\right.
$$

where $A=G_{111}, B=\frac{1}{6} G_{300}, C=\operatorname{Re} h_{110}, D=\frac{1}{2} \operatorname{Re} H_{210}, E=\frac{1}{2} \operatorname{Re} H_{021}$, and the $O$-terms depend on $(\eta, \rho, \theta)$. The coefficients in $(1.7)$ for $d=d^{*}$ have the following expressions

$$
\begin{aligned}
A^{*} & =-\frac{32 a_{1}^{3} a_{2} b_{1}^{3} b_{2}}{\left[4 a_{1} b_{1}+b_{2}\left(b_{1}+1\right)^{2}\right]^{3}}-\frac{8 a_{1}^{2} a_{2} b_{1}^{3}\left(b_{1}-1\right)}{\left(b_{1}+1\right)\left[4 a_{1} b_{1}+b_{2}\left(b_{1}+1\right)^{2}\right]^{2}}, \\
B^{*} & =-\frac{16 a_{1} a_{2}^{3} b_{1}^{3}\left(b_{1}+1\right)^{4}}{\left[4 a_{1} b_{1}+b_{2}\left(b_{1}+1\right)^{2}\right]^{4}}, \\
C^{*} & =\frac{2 a_{1} a_{2} b_{2}\left(b_{1}+1\right)^{2}}{\left[4 a_{1} b_{1}+b_{2}\left(b_{1}+1\right)^{2}\right]^{2}}-\frac{a_{2}\left(b_{1}^{2}-1\right)}{4 a_{1} b_{1}+b_{2}\left(b_{1}+1\right)^{2}},
\end{aligned}
$$




$$
\begin{aligned}
D^{*} & =\frac{16 a_{1} a_{2}^{3} b_{1}^{3}\left(b_{1}+1\right)^{4}}{\left[4 a_{1} b_{1}+b_{2}\left(b_{1}+1\right)^{2}\right]^{4}}-\frac{2 a_{2}^{2} b_{1}\left(b_{1}+1\right)^{3}}{\left[4 a_{1} b_{1}+b_{2}\left(b_{1}+1\right)^{2}\right]^{2}}, \\
E^{*} & =-\frac{a_{1} b_{1}\left(b_{1}-1\right)}{2\left(b_{1}+1\right)^{2}} .
\end{aligned}
$$

These formulae, first obtained by Klebanoff while developing his doctoral thesis, have been published (with some misprints) in [17]. It is worthwhile to point out that the coefficients in (1.7) are not uniquely defined. Indeed, one can multiply the eigenvector $q$ by a real number $\kappa \neq 0$ and the eigenvector $w$ by a complex number $\varepsilon \neq 0$, and rescale the adjont eigenvectors $p$ and $v$ to preserve relative normalization $\langle p, q\rangle=\langle v, w\rangle=1$. Then the coefficients $A, B, C, D$, and $E$ will change. The Klebanoff-Hastings expressions are obtained with the above choice of the eigenvectors.

Finally, a linear approximation to the function $\delta=\delta(d)$ near $d=d^{*}$ can be derived. Notice that $(\alpha, \beta)$ can be considered as new parameters near $d^{*}$, since the map $\left(d_{1}, d_{2}\right) \mapsto(\alpha, \beta)$, with $\alpha$ and $\beta$ given by (1.3) and (1.2) is regular at $d=d^{*}$. The Inverse Function Theorem then gives

$$
\begin{aligned}
& d_{1}=d_{1}^{*}-\frac{4 a_{1}}{b_{1}^{2}-1} \alpha+O\left(\|(\alpha, \beta)\|^{2}\right), \\
& d_{2}=d_{2}^{*}-\beta+O\left(\|(\alpha, \beta)\|^{2}\right) .
\end{aligned}
$$

The second component of the eigenvector $q$ can then be written in terms of $(\alpha, \beta)$ as

$$
q_{2}=\frac{a_{2} b_{1}\left(b_{1}+1\right)^{3}}{a_{1}\left(b_{1}-1\right)\left[4 a_{1} b_{1}+b_{2}\left(b_{1}+1\right)^{2}\right]}[2 \alpha-\beta]+O\left(\|(\alpha, \beta)\|^{2}\right),
$$

provided that $q_{1}=q_{1}^{*}$ for all $d$ close to $d^{*}$. Thus, taking into account (1.5),

$$
\delta=\frac{16 a_{1} a_{2}^{2} b_{1}^{3}\left(b_{1}+1\right)^{3}}{\left(b_{1}-1\right)\left[b_{2}\left(b_{1}+1\right)^{2}+4 a_{1} b_{1}\right]^{3}}[2 \alpha-\beta]+O\left(\|(\alpha, \beta)\|^{2}\right) .
$$

\subsection{Time reparametrization}

The normal form (1.7) can be further simplified by introducing a new time $\tau$ related to the original time $t$ by

$$
d t=(1+\mu \eta) d \tau
$$

where $\mu$ is a free parameter. In the new time, (1.7) takes the form

$$
\left\{\begin{array}{l}
\dot{\eta}=\beta \eta+(\delta+\mu \beta) \eta^{2}+(A+\mu \delta) \rho^{2} \eta+B \eta^{3}+O\left(\|(\eta, \rho)\|^{4}\right) \\
\dot{\rho}=\alpha \rho+(C+\mu \alpha) \eta \rho+(D+\mu C) \eta^{2} \rho+E \rho^{3}+O\left(\|(\eta, \rho)\|^{4}\right) \\
\dot{\theta}=\omega+O(\|(\eta, \rho)\|),
\end{array}\right.
$$

where dots now mean differentiation with respect to $\tau$. If $C^{*} \neq 0$, which is generically true, then the $\eta^{2} \rho$-term in the second equation can be annihilated by setting

$$
\mu=-\frac{D}{C} \text {. }
$$

The following simplified normal form is therefore obtained

$$
\left\{\begin{array}{l}
\dot{\eta}=\beta \eta+\gamma \eta^{2}+A_{1} \rho^{2} \eta+B \eta^{3}+O\left(\|(\eta, \rho)\|^{4}\right) \\
\dot{\rho}=\alpha \rho+C_{1} \eta \rho+E \rho^{3}+O\left(\|(\eta, \rho)\|^{4}\right) \\
\dot{\theta}=\omega+O(\|(\eta, \rho)\|)
\end{array}\right.
$$

where

$$
\gamma=\delta-\beta \frac{D}{C}, A_{1}=A-\delta \frac{D}{C}, C_{1}=C-\alpha \frac{D}{C} .
$$

Notice that $\gamma=0, A_{1}=A^{*}$ and $C_{1}=C^{*}$ at $d=d^{*}$. Moreover, the time reparametrization does not change the linear dependence of $\gamma$ upon $\alpha$ (see (1.8)). The normal form (1.9) coincides with (3.1).

\section{REFERENCES}


1. P.A. Abrams and J.D. Roth. The effects of enrichment of three-species food chains with nonlinear functional responses. Ecology, 75:1118-1130, 1994.

2. P.A. Abrams and J.D. Roth. The responses of unstable food chains to enrichment. Evol. Ecol., 8:150-171, 1994.

3. V.I. Arnold. Geometrical Methods in the Theory of Ordinary Differential Equations. SpringerVerlag, New York, Heidelberg, Berlin, 1983.

4. T.R. Chay. Chaos in a three variable model of an excitable cell. Physica D, 16:233-242, 1985.

5. K.S. Cheng. Uniqueness of a limit cycle of a predator-prey system. SIAM J. Math. Anal., 12:541$548,1981$.

6. H. El-Owaidy and A.A. Ammar. Mathematical analysis of a food-web model. Math. Biosci., 81:231-227, 1986.

7. H.I. Freedman and J.W.H. So. Global stability and persistence of simple food chains. Math. Biosci., 76:69-86, 1985.

8. H.I. Freedman and P. Waltman. Mathematical analysis of some three-species food-chain models. Math. Biosci., 33:257-276, 1977.

9. H.I. Freedman and P. Waltman. Persistence in models of three interacting predator-prey populations. Math. Biosci., 68:213-231, 1984.

10. T.C. Gard. Persistence in food webs: Holling-type food chains. Math. Biosci., 49:61-67, 1980.

11. T.C. Gard. Uniform persistence in multispecies population models. Math. Biosci., 85:93-104, 1987.

12. T.C. Gard and T.G. Hallam. Persistence in food-webs - I, Lotka-Volterra food chains. Bull. Math. Biol., 41:877-891, 1979.

13. J. Guckenheimer and Ph. Holmes. Nonlinear Oscillations, Dynamical Systems and Bifurcations of Vector Fields. Springer-Verlag, New York, Heidelberg, Berlin, 1983.

14. A. Hastings and T. Powell. Chaos in a three-species food chain. Ecology, 72:896-903, 1991.

15. P. Hogeweg and B. Hesper. Interactive instruction on population interactions. Comp. Biol. Med., 8:319-327, 1978.

16. A.I. Khibnik, Yu.A. Kuznetsov, V.V. Levitin, and E.V. Nikolaev. Continuation techniques and interactive software for bifurcation analysis of ODEs and iterated maps. Physica D, 62:360-371, 1993.

17. A. Klebanoff and A. Hastings. Chaos in three species food chains. J. Math. Biol., 32:427-451, 1994.

18. Yu.A. Kuznetsov. Elements of Applied Bifurcation Theory. Springer-Verlag, Berlin, Heidelberg, New York, 1995. (To appear).

19. Yu.A. Kuznetsov, S. Muratori, and S. Rinaldi. Homoclinic bifurcations in slow-fast second order systems. Nonlinear Analysis, 1995. (To appear).

20. R.M. May. Limit cycles in predator-prey communities. Science, 177:900-902, 1972.

21. K. McCann and P. Yodzis. Biological conditions for chaos in a three-species food chain. Ecology, 75:561-564, 1994.

22. K. McCann and P. Yodzis. Bifurcation structure of a three species food chain model. Theor. Pop. Biol., 48, 1995. (To appear).

23. S. Muratori. An application of the separation principle for detecting slow-fast limit cycles in a three-dimensional system. Appl. Math. Comp., 43:1-18, 1991.

24. S. Muratori and S. Rinaldi. Catastrophic bifurcations in a second-order dynamical system with applications to acid rain and forest collapse. Appl. Math. Modelling, 13:67-681, 1989.

25. S. Muratori and S. Rinaldi. A dynamical system with Hopf bifurcations and catastrophes. Appl. 
Math. Comp., 29:1-15, 1989.

26. S. Muratori and S. Rinaldi. A separation condition for the existence of limit cycles in slow-fast systems. Appl. Math. Modelling, 15:312-318, 1991.

27. S. Muratori and S. Rinaldi. Low- and high-frequency oscillations in three-dimensional food chain systems. SIAM J. Appl. Math., 52:1688-1706, 1992.

28. C. Piccardi, G. Feichtinger, and C.V. Forst. A nonlinear dynamical model for the dynastic cycle. Chaos, Solitons and Fractals, 1995. (To appear).

29. V. Rai and R. Sreenivasan. Period-doubling bifurcations leading to chaos in a model food chain. Ecol. Modelling, 69:63-77, 1993.

30. A. Rescigno and K.G. Jones. The struggle for life: III. A predator-prey chain. Bull. Math. Bioph., 34:521-532, 1972.

31. M. Scheffer. Should we expect strange attractors behind plankton dynamics and if so, should we bother? J. Plankton Res., 13:1291-1305, 1991.

32. M. Scheffer. Fish and nutrients interplay determines algal biomass. Oikos, 62:271-282, 1992.

33. A. Sherman, J. Rinzer, and J. Keuzer. Emergence of organized bursting in clusters of pancreaic $\alpha$-cells by channel sharing. Biophys. J., 54:411-425, 1988.

34. D. Terman. Chaotic spikes arising from a model for bursting in excitable membranes. SIAM J. Appl. Math., 51:1418-1450, 1991.

35. D. Terman. The transition from bursting to continuous spiking in excitable mambrane models. J. Nonlin. Sci., 2:135-182, 1992.

36. J.W. Wilder, N. Voorhis, J.J. Colbert, and A. Sharov. A three variable differential equation model for gypsy moth population dynamics. Ecol. Modelling, 72:229-250, 1994. 